\title{
Initial Cavity Ring-Down Density Measurement on a 6-kW Hall Thruster
}

\author{
Wensheng Huang ${ }^{*}$, Alec D. Gallimore ${ }^{\dagger}$, and Timothy B. Smith ${ }^{\ddagger}$ \\ Plasmadynamics and Electric Propulsion Laboratory, University of Michigan, Ann Arbor, MI 48109 \\ and \\ Lei $\mathrm{Tao}^{\S}$ and Azer P. Yalin ${ }^{* *}$ \\ Laser Plasma Diagnostics Laboratory, Colorado State University, Fort Collin, CO 80521
}

\begin{abstract}
We present the initial results from a cavity ring-down sensor for measuring the density of sputtered boron atoms originating from the discharge channel of a $6-\mathrm{kW}$ Hall thruster. The sensor traps $250 \mathrm{~nm}$ light in a high-finesse cavity to greatly increase its sensitivity to boron atoms. Measurements were obtained with the thruster operating at seven conditions spanning discharge voltages from 150 to $600 \mathrm{~V}$, and anode mass flow rates from 10 to 30 $\mathrm{mg} / \mathrm{s}$. Power level at these operating conditions ranged from 1.5 to $10 \mathrm{~kW}$. Boron density was found to vary from $<1 \times 10^{14}$ to $\sim 1 \times 10^{15} \mathrm{~m}^{-3}$ with the higher density measurements being found near the two channel walls. We apply a simple two-dimensional velocity model to obtain boron flux. The boron flux is then correlated to the boron nitride sputter rate, which is found to vary from $4 \times 10^{-4}$ to $6 \times 10^{-3} \mathrm{~mm}^{3} / \mathrm{s}$. A simple power law equation is formulated to correlate the relative sputter rate for the 6-kW Hall thruster as a function of the tested operating conditions. Although the uncertainty in this initial result is high, the result clearly demonstrates previously untapped potential for the use of cavity ring-down spectroscopy to study the problem of Hall thruster channel wall erosion.
\end{abstract}

\section{Introduction}

A LTHOUGH Hall thruster is rapidly maturing as a propulsion technology in terms of performance, there is a lack of understanding of the physics that limits the lifetime. In particular, the physics behind the erosion of the ceramic channel walls remains an active area of research. Our understanding of this erosion phenomenon is limited in large part due to the difficulties associated with obtaining parametric experimental data using traditional approaches. We present the initial results of a novel approach that uses a cavity ring-down sensor to detect sputtered particle density in near-real-time as well as methods for correlating the results to erosion rates.

The simplest approach to measuring channel wall erosion rate is to run the thruster for a set number of hours and measure the channel wall profile via profilometry. ${ }^{1,2}$ Other potential channel erosion diagnostics include quartz crystal microbalance ${ }^{3,4}$ and emission spectroscopy. ${ }^{3,5}$ Weight loss is also regularly used to measure sputter yield. ${ }^{6-8}$ Profilometry require extended run times (weeks to months) to obtain accurate results, which translates to large amount of resources and time spent. Parametric studies for these two diagnostics are very difficult for any but the largest institutions to carry out and the test matrix must be picked out carefully beforehand with little margin for error. Quartz crystal microbalance (QCM) has the potential to obtain differential sputtering profile in tens of hours to days but has many technical issues associated with thermal stabilization and etching when applied in a Hall thruster environment. ${ }^{3}$ It is also limited to far-field interrogation and gives little information about what is happening near the erosion sites. Optical emission spectroscopy (OES) is another technique that can obtain erosion data in hours to days but is usually limited to relative density measurements. The accuracy of OES is generally not very

\footnotetext{
*Ph. D. Candidate, Aerospace Engineering, davhuang@ umich.edu, AIAA Student Member.

${ }^{\dagger}$ Arthur F. Thurnau Professor, Aerospace Engineering, alec.gallimore@ umich.edu, AIAA Fellow.

${ }^{\ddagger}$ Lecturer, Aerospace Engineering, timsmith@umich.edu, AIAA Senior Member.

${ }^{\S}$ Ph. D., Mechanical Engineering, leitao@lamar.colostate.edu, AIAA Member.

** Associate Professor, Mechanical Engineering, ayalin@engr.colostate.edu, AIAA Senior Member.
}

1

American Institute of Aeronautics and Astronautics 
high because it requires accurate knowledge of the state of the plasma species in the region where the measurement is taken.

Cavity ring-down spectroscopy (CRDS) provides a novel solution to the problem of obtaining parametric experimental sputter rate data. This technique is non-intrusive, spatially resolved in the radial direction, and capable of measuring absolute density with extreme sensitivity. The time scale for deploying CRDS diagnostic is on the order of hours to days. For this technique, laser light trapped in a high-finesse cavity is resonantly absorbed by erosion products. Since the trapped laser light makes many passes through the test sample, CRDS has an exceptionally high sensitivity to even minute amounts of erosion products. Since the measurement results yield lineintegrated product density, some form of inversion is needed to obtain radial density profiles. One can then combine the CRDS density results with velocity results from modeling or a velocimetry technique like laser-induced fluorescence (LIF) to obtain the sputtering flux. Alternately, one can perform control experiments to correlate product density to sputter yield given a priori knowledge about the bombarding particles. The main disadvantages of CRDS are the high initial cost of associated equipment relative to more traditional diagnostics, the complexity of the setup, and the associated implementation challenges. However, CRDS can yield tremendous long-term savings in resource by greatly reducing the thruster and facility run time needed to obtain data of fidelity comparable to traditional diagnostics.

The history of the use of CRDS in Hall thruster-related research is fairly short. The first work investigating the possibility of the use of CRDS for studying erosion in electric propulsion devices was by Surla, et al., in 2004. ${ }^{9}$ The initial focus was on anode layer Hall thrusters ${ }^{10}$ which have metallic wall. Metallic elements have transition lines that were easier to access than the ceramic elements found in the walls of magnetic layer thrusters. To study the application of CRDS for magnetic layer type thrusters, which are much more common in the U.S. than anode layer type, a collaborative program between the University of Michigan and Colorado State University was initiated. Initial work focused on the feasibility of generating, delivering, and trapping $250 \mathrm{~nm}$ light for probing boron, as well as the use of continuous-wave-CRDS over pulsed-CRDS to enhance sensitivity. ${ }^{11,}{ }^{12}$ At the same time, basic research work was carried out on the sputtering behavior of boron nitride when bombarded by low-energy xenon. ${ }^{4}$ The said collaborative research program culminated in the first published demonstration of the use of CRDS to measure the channel wall erosion rate in a magnetic layer Hall thruster, which is described in this paper.

\section{Theories}

\section{A. Principles of Cavity Ring-Down Spectroscopy}

Cavity ring-down spectroscopy is a type of absorption spectroscopy. A great review of the topic and recent development of CRDS as a diagnostic can be found in an article by Berden, et al. ${ }^{13}$ CRDS is based on the principle that by sending a beam of photons through an absorber and measuring the change in intensity, one can obtain the absolute line-integrated density of the absorber. CRDS differs from traditional absorption spectroscopy in that a cavity, formed from a pair of highly reflective mirrors, is used to trap light. Instead of one pass, the trapped photons make many passes through the absorbing medium. Each time the trapped light strikes one of the mirrors, a small fraction of it leaks out. The intensity of the leaked light is measured by a detector. If the light source is a continuouswave laser, the laser beam is cut off rapidly (e.g. with an acousto-optical modulator), and then the light intensity within the cavity begin to decay. The characteristic time constant of the decay, extracted from the detector signal, can then be used to calculate the absolute line-integrated density of the absorber inside the cavity. Figure 1 illustrates the operational principles of CRDS. The grey arrow pointing from the detector to the acousto-optical modulator (AOM) indicates some form of feedback so the AOM knows when to cut off the laser beam.

Equations (1) and (2) govern the ring-down behavior and time constant in a typical cavity,

$$
\mathrm{S}(\mathrm{t}, \mathrm{v})=\mathrm{S}_{0} \exp [-\mathrm{t} / \tau(\mathrm{v})]
$$

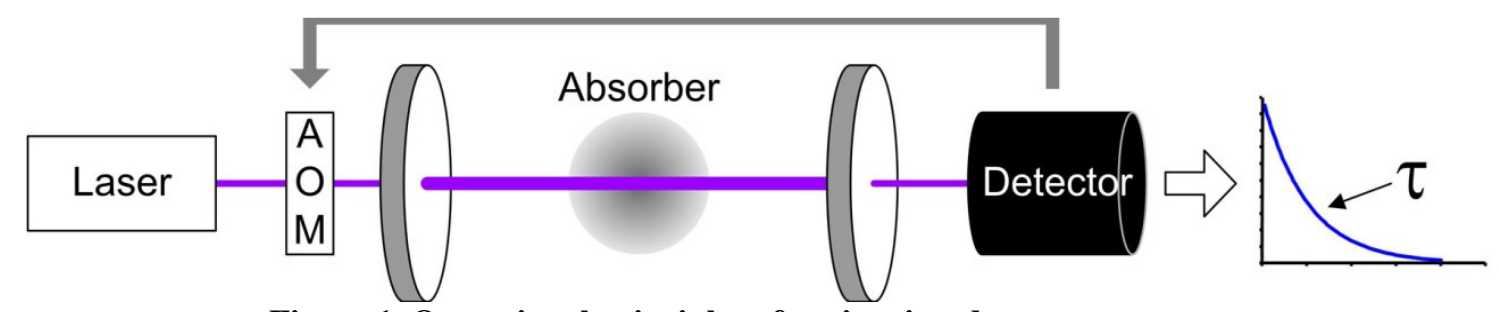

Figure 1. Operational principles of cavity ring-down spectroscopy.

American Institute of Aeronautics and Astronautics 


$$
1 / \tau(v)=\frac{c}{L}\left[\int k(x, v) d x+\left(1-R_{M}\right)\right]
$$

where $S$ is the signal intensity, $S_{0}$ is the initial signal intensity, $\tau$ is the characteristic time of decay (also called the ring-down time), $\mathrm{c}$ is the speed of light, $\mathrm{L}$ is the cavity length defined as the distance between the two mirrors, and $\mathrm{R}_{\mathrm{M}}$ is the mirror reflectivity. $\mathrm{k}(\mathrm{x}, \mathrm{v})$ is the absorption coefficient at a given laser frequency, $\mathrm{v}$, and position along the cavity optical axis, $x$. The integral term represents the line-integrated contribution from the absorber. In practice, the mirror reflectivity changes in time and there is a scattering effect that decreases the effective reflectivity. To extract absolute line-integrated density, the ring-down time is compared to the empty cavity ring-down time, $\tau_{0}$. This is shown in Eq. (3),

$$
\operatorname{Abs}(v) \equiv \int k(x, v) d x=\frac{L}{c}\left[\frac{1}{\tau(v)}-\frac{1}{\tau_{0}}\right]
$$

where Abs is the sample absorbance and is unitless. By scanning the laser frequency across an entire transition line, one can then integrate for the total absorbance and relate it back to the absolute density of the lower state of the transition being studied. Equation (4) shows this relationship,

$$
\int n_{1} d x=8 \pi \frac{g_{1}}{g_{2}} \frac{v_{21}{ }^{2}}{A_{21} c^{2}} \int \operatorname{Abs}(v) d v
$$

where $\mathrm{n}$ is absolute density, $\mathrm{g}$ is level degeneracy, $\mathrm{v}$ is transition frequency, $\mathrm{A}$ is the spontaneous emission rate, subscript 1 denotes lower state, and subscript 2 denotes upper state. Table 1 lists the values of the various constants for the $249.848 \mathrm{~nm}$ transition.

A typical Hall thruster can usually be assumed to be axisymmetric (there are cases where this is not true, e.g. when an external cathode perturbs the axisymmetry ${ }^{15}$ ). To obtain erosion product density as a function of radial position, we begin by taking CRDS density measurements across multiple $\mathrm{P}$ positions. Here, the P-axis is orthogonal to the laser beam axis and parallel to the channel exit plane of the thruster. Figure 2 illustrates the geometry of the Paxis. Note that due to the way $\mathrm{P}$ is non-dimensionalized, $\mathrm{P}=0$ correspond to the nominal inner wall radius of the thruster and not the center of the thruster. The density measurements can then be converted to a function of radial position via some form of inversion method like the onion-peeling method ${ }^{16}$ or the Abel inversion method. ${ }^{17}$ The onion-peeling method is selected for the current work because it is mathematically much simpler than Abel inversion for a discretized data set of relatively small size. A comparison of various inversion methods including onion-peeling and Abel can be found in a paper by Dasch. ${ }^{16}$ Detailed description of the onion-peeling method is presented in a later section.

The cavity used in CRDS is also called a resonator because only light of the right frequencies can resonate with the cavity and build up in the cavity. In the simplest analysis, the governing equation for the propagating frequencies in a planar cavity can be found by trying to fit a standing wave into the cavity. Since the mirrors are near lossless, the boundary condition at the mirror must correspond to a nodal point in the standing wave. These conditions give rise to Eq. (5), ${ }^{18}$

$$
v_{q}=q \frac{c}{2 L}, q=1,2,3, \ldots
$$

where $v_{\mathrm{q}}$ is the frequency of the q-th resonance mode and can only be certain discrete values in a perfectly lossless

Table 1. Transition parameters for the B I $249.848 \mathrm{~nm}$ transition. ${ }^{14}$

\begin{tabular}{cc}
\hline Constant & Value \\
\hline $\mathbf{g}_{1}$ & 4 \\
$\mathbf{g}_{\mathbf{2}}$ & 2 \\
$\mathbf{v}_{\mathbf{2 1}}$ & $1.19990 \mathrm{e} 15 \mathrm{~Hz}$ \\
$\mathbf{A}_{\mathbf{2 1}}$ & $1.67 \mathrm{e} 8 \mathrm{~s}^{-1}$ \\
\hline
\end{tabular}

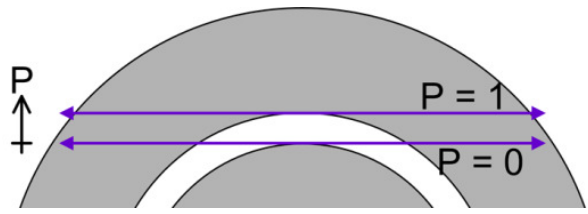

Figure 2. Diagram illustrating the geometry of the P-axis. 
cavity. In a lossy cavity, transmission can happen over a narrow range of frequencies centered on each resonance frequency. The FWHM value of each transmission peak is described by Eqs. (6) to (8), ${ }^{18}$

$$
\begin{gathered}
\delta v \approx \Delta v_{\mathrm{F}} / \underline{F} \\
\underline{\mathrm{F}}=\frac{\pi \sqrt{\mathrm{R}_{\mathrm{M}}}}{1-\mathrm{R}_{\mathrm{M}}} \\
\Delta v_{\mathrm{F}}=\frac{\mathrm{c}}{2 \mathrm{~nL} \cos \theta} \approx \frac{\mathrm{c}}{2 \mathrm{~L}}
\end{gathered}
$$

where $\delta v$ is the spectral width of the individual resonance mode, $\Delta v_{\mathrm{F}}$ is the frequency spacing between resonance peaks (also called the free spectral range), $\underline{F}$ is the cavity finesse, $R_{M}$ is the mirror reflectivity, $n$ is the refractive index of the medium between the mirrors, and $\theta$ is the angle of incidence with respect to the mirror surface normal. Both $\mathrm{n}$ and $\cos \theta$ are typically equal to 1 . From these equations we can see that the finesse increases rapidly as reflectivity approaches 1 . Note that $R_{M}=1$ represents a lossless mirror. From Eq. (2), we see that we want the mirror to have a reflectivity as close to 1 as possible because then the $\left(1-\mathrm{R}_{\mathrm{M}}\right)$ term becomes negligible and the ring-down time is sensitive only to the absorber. Furthermore, as the finesse increases, the spectral width decreases and one can obtain higher resolution scans.

Our approach for BN erosion rate measurement is to scan the ground state boron transition at $249.848 \mathrm{~nm}$ to measure boron density. For this wavelength, commercial mirrors can achieve a typical reflectivity of $\sim 0.997$, which corresponds to a finesse of $\sim 1000$. This reflectivity is poor compared to that available for reflectors in the visible and infrared, which makes sensitive detection challenging compared to standard CRDS. Equation (9) shows the two primary attributes of a CRDS sensor that determines its minimum detection threshold. ${ }^{13}$

$$
[\operatorname{Abs}(v)]_{\min }=\left(1-\mathrm{R}_{\mathrm{M}}\right)\left(\frac{\Delta \tau}{\tau}\right)_{\min }
$$

where $\Delta \tau$ is the uncertainty in the measured ring-down time. To improve the sensor's sensitivity, one can either increase the mirror reflectivity or decrease the ring-down time measurement uncertainty. For a cavity length of $\sim 1$ $\mathrm{m}, \Delta v_{\mathrm{F}}$ equals $150 \mathrm{MHz}$, so $\delta v$ equals $150 \mathrm{kHz}$. It is difficult for a commercial laser to achieve a frequency width that narrow (typical diode laser can achieve $\sim 1 \mathrm{MHz}$ ), so only a portion of the input laser beam power couples into the cavity. Increasing mirror reflectivity increases measurement sensitivity but can also decrease the beam power that can be coupled into the cavity, which decreases the signal. Furthermore, the mirror reflectivity, the laser power, and the laser linewidth are limited by technology. Alternately, one can improve the CRDS sensor's sensitivity by decreasing the ring-down time measurement uncertainty. This uncertainty is limited by the amount of signal compared to the amount of noise, and the size of the data sample. Typically, the sample size is the easiest to influence and is determined by the ring-down rate, the sampling rate, and the sampling time.

The governing equations for cavity transmission shown in this work are for the $\mathrm{TEM}_{\mathrm{q} 00}$ modes, which are called the fundamental modes. Other non-fundamental modes of transmission are also possible and require waveoptics treatment of the problem. ${ }^{18}$ For our purpose, we want to couple light into only the fundamental modes because higher peak intensity can be achieved with those modes than non-fundamental modes and the sets of modes have different ring-down time. To be more precise, we are coupling into only one of the fundamental modes at any given time because Eq. (5) can only be satisfied for one value of $q$ if the laser is monochromatic. Thus, the $\mathrm{TEM}_{\mathrm{q} 00}$ modes are often collectively referred to as the $\mathrm{TEM}_{00}$ mode. The process of coupling light into only the TEM $\mathrm{T}_{00}$ mode is called mode-matching, the theory of which is described in work by Kogelnik and $\mathrm{Li}^{19}$ 


\section{B. Onion Peeling}

The onion-peeling method is based on the idea that one can divide the axisymmetric data zone into concentric rings of constant property. The distance that the CRDS cavity beam travels through each of these concentric rings can be calculated by geometry. A matrix can then be formed that relates the measured line-integrated density to the density within each ring. Figure 3 illustrates this concept. This method is intuitively simple and computationally inexpensive when the number of data points is relatively low. The matrix formed for this method is an upper triangular matrix and the solution can be obtained by Gaussian elimination. Equation (10) shows the matrix representation of the onion-peeling method.

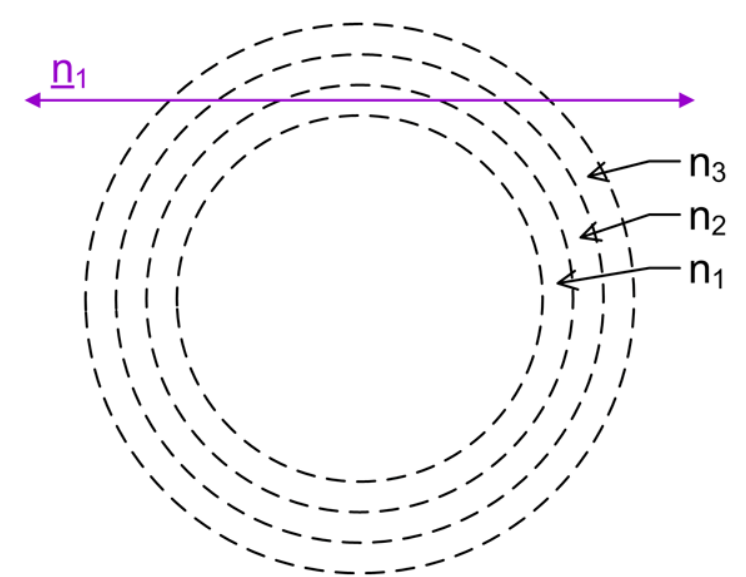

Figure 3. Illustration for the onion-peeling concept.

$$
\mathbf{K} \cdot \mathbf{n}=\underline{\mathbf{n}}
$$

where $\mathbf{K}$ is the geometry matrix and is upper triangular, $\mathbf{n}$ is the density column vector, and $\underline{\mathbf{n}}$ is the line-integrated density column vector. Equation (11) shows the formula that governs how $\mathbf{K}$ is calculated.

$$
\mathrm{K}_{\mathrm{ji}}= \begin{cases}0, & \mathrm{i}<\mathrm{j} \\ 2 \sqrt{\mathrm{r}_{\mathrm{i}}{ }^{2}-\mathrm{p}_{\mathrm{j}}{ }^{2}}, & \mathrm{i}=\mathrm{j} \\ 2 \sqrt{\mathrm{r}_{\mathrm{i}}{ }^{2}-\mathrm{p}_{\mathrm{j}}{ }^{2}}-2 \sqrt{\mathrm{r}_{\mathrm{i}-1}{ }^{2}-\mathrm{p}_{\mathrm{j}}{ }^{2}}, & \mathrm{i}>\mathrm{j}\end{cases}
$$

where the subscript $i$ is both the column index of $\mathbf{K}$ and the position index for $r$, the radial coordinate in dimensional form, and the subscript $\mathbf{j}$ is both the row index of $\mathbf{K}$ and the position index for $\mathrm{p}$, which is the vertical coordinate and the dimensional form of $\mathrm{P}$ from Fig. 2. Note that $\mathrm{r}$ and $\mathrm{p}$ have a one-to-one correspondence at the $12 \mathrm{o}$ 'clock position of the thruster when viewed face-on. Also, the position $r_{i}$ is associated with the density $n_{i}$ while the position $p_{j}$ is associated with the line-integrated density $\underline{\underline{n}}_{\text {. }}$. More details regarding the mathematical implementation of the onionpeeling method can be found in a paper by Dasch. ${ }^{16}$

\section{Sigmund Thompson Distribution}

The velocity distribution function of sputtered particles from bombardment of single-component target is a wellstudied subject. This distribution function is described by the Sigmund-Thompson distribution, ${ }^{20,}{ }^{21}$ shown in Eq. (12) and (13),

$$
\begin{gathered}
f(u) \sim \frac{u^{3}}{\left(u^{2}+v_{b}{ }^{2}\right)^{3-2 m}} \cos \phi \\
v_{b}=\sqrt{\frac{2 E_{b}}{M}}
\end{gathered}
$$

where $\mathrm{u}$ is the velocity, $\mathrm{v}_{\mathrm{b}}$ can be calculated from the binding energy, $\mathrm{E}_{\mathrm{b}}$, using Eq. (13), $\phi$ is the polar angle of the emitted particle with respect to the surface normal, $\mathrm{m}$ is an energy-dependent parameter and is close to 0 for low inbound energy $(<1 \mathrm{keV})$, and $\mathrm{M}$ is the mass of the sputtered particle. The binding energy is a constant that depends on the material and the surface condition. This distribution is insensitive to bombardment angle and not very sensitive to bombardment energy at low bombardment energy $(<1 \mathrm{keV})$. Note that the Sigmund-Thompson distribution function show in Eq. (12) is flux-based as oppose to number density-based. 
For a typical magnetic-layer Hall thruster, the channel walls are made of boron nitride, which is a multi-component material. Active research is being carried out at Colorado State University to determine how sputtered boron behaves when boron nitride is bombarded by low energy xenon. ${ }^{22}$ Preliminary work by Tao and Yalin shows that for xenon bombardment energy of $1200 \mathrm{eV}$, a Sigmund-Thompson distribution with $\mathrm{m}=0.17 \pm 0.05$ and $\mathrm{E}_{\mathrm{b}}=5.0 \pm 0.5 \mathrm{eV}$ fits the VDFs obtained in a controlled environment via LIF velocimetry very well. ${ }^{22}$ However, it is also pointed out in the same work that the value of the fitted binding energy is very sensitive to the value of $\mathrm{m}$, and that an $\mathrm{m}$ of $0-0.3$ all produce reasonable fit to the data. Furthermore, the average velocity of the Sigmund-Thompson distribution is also very sensitive to $\mathrm{m}$. If we use the limit at which the SigmundThompson distribution is supposed to be applicable as the limit of integration, the numerically integrated average

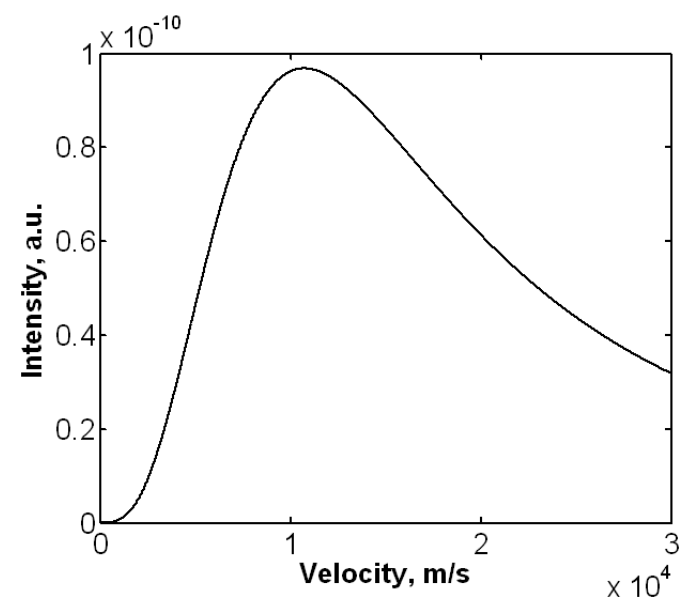

Figure 4. Example Sigmund-Thompson distribution with $\mathrm{m}=0.17, \mathrm{~Eb}=5 \mathrm{eV}$. velocity of the $\mathrm{m}=0.17, \mathrm{E}_{\mathrm{b}}=5 \mathrm{eV}$ distribution is $\sim 23 \mathrm{~km} / \mathrm{s}$. The aforementioned limit is described by Eq. (14), ${ }^{21}$

$$
\mathrm{T}_{\max }=\frac{4 \mathrm{M}_{1} \mathrm{M}_{2}}{\left(\mathrm{M}_{1}+\mathrm{M}_{2}\right)^{2}} \mathrm{E}_{\mathrm{ion}}
$$

where $T_{\max }$, the limit, is also the maximum energy transferred in an elastic head-on collision, $\mathrm{M}_{1}$ is the mass of the bombarding particle, $\mathrm{M}_{2}$ is the mass of the sputtered particle, and $\mathrm{E}_{\mathrm{ion}}$ is the energy of the bombarding particle. The last variable has the subscript "ion" because sputtering experiments are typically performed with mono-energetic ion beams.

Figure 4 shows an example Sigmund-Thompson distribution with $\mathrm{m}=0.17, \mathrm{E}_{\mathrm{b}}=5 \mathrm{eV}$. In the Hall thruster environment, the energy of the bombarding particles range from zero to a few hundred eV. Whether the sputtered particle distribution function still follows the Sigmund-Thompson distribution is an open area of research. The current hypothesis is that they do based on theories and observed behaviors from the bombardment of singlecomponent metallic targets. $^{21}$

Since some of the boron that exits the channel will also carry a radial component, the CRDS result, which is obtained from shooting a laser beam across the channel exit of the thruster, will be Doppler broadened. The exact amount of Doppler broadening is an open topic of research, and is expected to be no greater than the spread in the Sigmund-Thompson distribution. This value can potentially range from tens of $\mathrm{GHz}$ to hundreds of $\mathrm{GHz}$ depending on the exact angle at which the sputtered boron is traveling with respect to the CRDS laser beam.

\section{Experimental Setup}

\section{A. Thruster}

The 6-kW annular Hall thruster nominally operates at $300 \mathrm{~V}$ discharge voltage and $20 \mathrm{mg} / \mathrm{s}$ anode mass flow rate. Table 2 lists the seven operating conditions tested during this experiment ("Misc" is not a tested operating condition). Note that the discharge voltage and anode mass flow rate are the main varying parameters between conditions. For the rest of this paper, operating conditions are labeled as YYY V, ZZ mg/s, where YYY is the discharge voltage and $\mathrm{ZZ}$ is the anode mass flow rate. Cathode mass flow rate is fixed to $7 \%$ of the anode mass flow rate for all operating conditions. Magnetic field topology is a magnetic lens
Table 2. List of operating conditions

\begin{tabular}{cccc}
\hline $\begin{array}{c}\text { Discharge } \\
\text { voltage, } \mathbf{~}\end{array}$ & $\begin{array}{c}\text { Anode mass } \\
\text { flow rate, mg/s }\end{array}$ & $\begin{array}{c}\text { Discharge } \\
\text { Current, A }\end{array}$ & $\begin{array}{c}\text { Percentage } \\
\text { of run time }\end{array}$ \\
\hline 150 & 10 & 9.08 & $7.2 \%$ \\
150 & 20 & 21.55 & $5.8 \%$ \\
150 & 30 & 35.35 & $5.9 \%$ \\
300 & 10 & 8.94 & $29.4 \%$ \\
300 & 20 & 20.11 & $28.7 \%$ \\
300 & 30 & 33.25 & $9.5 \%$ \\
600 & 10 & 9.36 & $7.8 \%$ \\
Misc. & & & $5.7 \%$ \\
\hline
\end{tabular}


symmetric about the thruster channel centerline. Magnetic field magnitude is set to maximize thruster efficiency. These settings were obtained through the use of an inverse pendulum thrust stand.

At the conclusion of the CRDS test described in this paper, the 6-kW Hall thruster has been operated for a total of 543 hours. Table 2 shows the percentage of run time for each tested operating condition. The miscellaneous entry in the table includes operating conditions spanning discharge voltages from $105 \mathrm{~V}$ to $550 \mathrm{~V}$ and anode mass flow rates from $5 \mathrm{mg} / \mathrm{s}$ to $17.5 \mathrm{mg} / \mathrm{s}$. Profilometry measurement was taken at this point in time using a Brown \& Sharpe MicroVal 343 coordinate measuring machine.
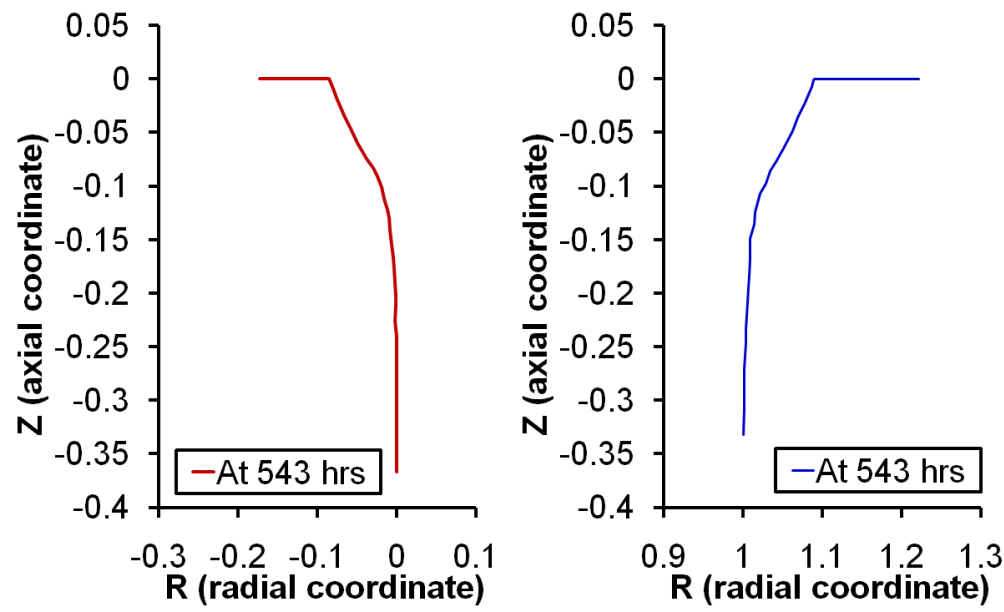

Figure 5. Profilometry measurements for the channel inner wall (left) and outer wall (right).

Figure 5 shows the measured channel profiles.

\section{B. Facility and Test Matrix}

Experiments were performed in the Large Vacuum Test Facility (LVTF) of the Plasmadynamics and Electric Propulsion Laboratory (PEPL) at the University of Michigan. The LVTF is a $\varnothing 6 \mathrm{~m} \times 9 \mathrm{~m}$ stainless steel-clad cylindrical iron chamber. The thruster plume expands un-impeded until termination at a beam dump $\sim 4 \mathrm{~m}$ downstream. Pumping is provided by seven cryopumps with a nominal xenon pumping speed of $245,0001 / \mathrm{s}$. Facility pressure is monitored by two hot-cathode ionization gauges. The base pressure is approximately $2 \times 10^{-7}$ Torr. The pressure during thruster operation at $30 \mathrm{mg} / \mathrm{s}$ anode mass flow rate is approximately $2 \times 10^{-5}$ Torr, corrected for xenon.

During the experiments, the 6-kW Hall thruster was mounted on an Aerotech motion stage that has $\sim 2 \mathrm{~m}$ of vertical travel and a positioning accuracy of $\pm 0.1 \mathrm{~mm}$. The absolute position is monitored by an incremental encoder. The optical cavity was fixed to the chamber floor.

Research-grade xenon propellant $(99.999 \%$ pure) was supplied to the thruster by commercially available flow meters and controllers, having an accuracy of $\pm 1 \%$. Calibration of the flow system was done by the constant volume method taking into account the effects of compressibility.

The data locations for the CRDS test will be reported using the non-dimensionalized coordinate P. The radial position is reported using the non-dimensionalized coordinate $\mathrm{R}$, where $\mathrm{R}=0$ at the nominal inner channel wall radius and $\mathrm{R}=1$ at the nominal outer channel wall radius. At the 12 o'clock azimuthal location when viewing the thruster face-on, the values of $\mathrm{P}$ are the same as the values of the non-dimensional radial coordinate $\mathrm{R}$. In other words, $\mathrm{P}=0$ when the CRDS laser beam is directly in front of the nominal inner channel radius at the 12 o'clock position and $\mathrm{P}=1$ when the beam is directly in front of the nominal outer channel radius at the 12 o'clock position. Figure 2 illustrates this concept.

The locations at which data traces were taken for all operating conditions range from $\mathrm{P}=-0.2$ to $\mathrm{P}=1.2$ at an interval of 0.1 for a total of 15 locations. Additional traces are taken at $\mathrm{P}=2$, which is sufficiently far away from the thruster plume along the channel exit plane that there should be no boron signal. A trace is a series of ring-down time measurements taken over a range of wavelengths. It is graphically represented as the ring-down time plotted against the wavelength. Thus, if there is no boron signal, the trace is a horizontal line. Taking $\mathrm{P}=2$ traces help the user check the alignment of the cavity. The $\mathrm{P}=2$ traces are taken at least once prior to the start of a data set and once afterward for a given operating condition. If both traces look like horizontal lines, then the cavity was assumed to be aligned during the acquisition of the entire data set.

At each data location, 101 wavelength values are scanned to construct the shape of the B I $249.848 \mathrm{~nm}$ transition. The total scan range is $70 \mathrm{GHz}$ centered on the transition. The ring-down time obtained at each wavelength is an average of 150 ring-downs, though the exact number can vary from 100 to 200 depending on how many ring-downs were deemed necessary to obtain good statistics.

\section{Air-Side Setup}


Figure 6 shows the air-side experimental setup for the CRDS study. The DAQ computer, labeled as DAQ PC, uses a custom Labview v9 program along with some manufacturer software to control various components. The DAQ PC communicates through a USB hub (not shown in figure) with four TST001 stepper motor controllers that control the mirror motors on the cavity. Through an Ethernet hub, the DAQ PC communicates with the Aerotech Ensemble MP10 vertical stage controller via the software Motion Composer v3 from Aerotech. Through the same Ethernet hub, the DAQ PC communicates with a laptop computer inside of the laser room, which is the enclosure on the left side of Fig. 6. The communication between the two computers is handled by standard Ethernet protocols programmed into custom Labview programs. Housing the UV laser in the laser room is necessary to protect the laser from the dust and vibration in the rest of the facility.

The laptop computer inside of the laser room controls a Measurement Computing USB-3101 analog output device, which controls the scanning voltage of the piezoelectric element in the laser. There is an additional RC lowpass filter (not shown) between the analog output device and the UV laser controller. This filter is designed to filter out as much high frequency noise as possible while maintaining $\sim 0.1 \mathrm{~s}$ rise time. The reason such a complicated setup was used to control the UV laser is because the distance between the DAQ PC to the UV laser controller is long enough that the signal line suffers from electromagnetic interference. The amount of noise is actually very small, but large enough to influence the stability of the second frequency-doubling stage of the laser. If the electronic noise in the scan control signal is too high, the laser power randomly drops to zero. The solution to this problem is to use the laptop computer as a communication relay. Since the laptop computer uses an in-line regulated power source (i.e. wall power goes to battery, then battery power goes to laptop), its USB output exhibits very low noise level. Combined with the RC low-pass filter, the fluctuation in the scan control signal is small enough to be barely noticeable in the final output power of the UV laser.

The UV laser is a Toptica TA-FHG-110 frequency-quadrupled diode laser. The laser is composed of three major components. The first component is a taper-amplified diode laser that typically outputs $\sim 500 \mathrm{~mW}$ of 1000 -nm light. The second component is a frequency doubling cavity in a bow-tie configuration that takes the 1000-nm light and frequency-doubles it to $\sim 170 \mathrm{~mW}$ of $500-\mathrm{nm}$ light. The third component is another frequency doubling cavity in a bow-tie configuration that frequency-doubles the $500-\mathrm{nm}$ light to $\sim 10 \mathrm{~mW}$ of $250-\mathrm{nm}$ light. The average linewidth of the 250-nm laser output is $\sim 4 \mathrm{MHz}$.

A small amount of the $\sim 1000 \mathrm{~nm}$ laser light generated by the taper-amplified diode laser is split off and outputted in a port separate from the main output. This infrared beam is coupled into a High Finesse WS-7 Fizeautype wavemeter, which has an accuracy of $\pm 60 \mathrm{MHz}$ at $1000 \mathrm{~nm}$, or $\pm 0.2 \mathrm{pm}$. The wavelength of the frequencyquadrupled light is calculated from this reading by simply dividing by 4 , which gives an accuracy of $\pm 0.05 \mathrm{pm}$ at $250 \mathrm{~nm}$, or $\pm 240 \mathrm{MHz}$ in frequency space. The wavemeter also has a resolution of $10 \mathrm{MHz}$ and updates at a rate of up to $150 \mathrm{~Hz}$. It communicates with the DAQ PC via a powered USB hub instead of directly due to the physical distance to the DAQ PC. During the CRDS test, to excite resonance in the CRDS cavity, the UV laser is continuously executing triangular sweeps at a rate of $\sim 1 \mathrm{pm} / \mathrm{s}$ over a range of $\sim 1 \mathrm{pm}$. This wavelength sweeping normally generates an oscillatory reading in the wavemeter, but the wavemeter software is set to perform floating average of 50 measurements so the actual value recorded into the raw CRDS data is a time-averaged value of the

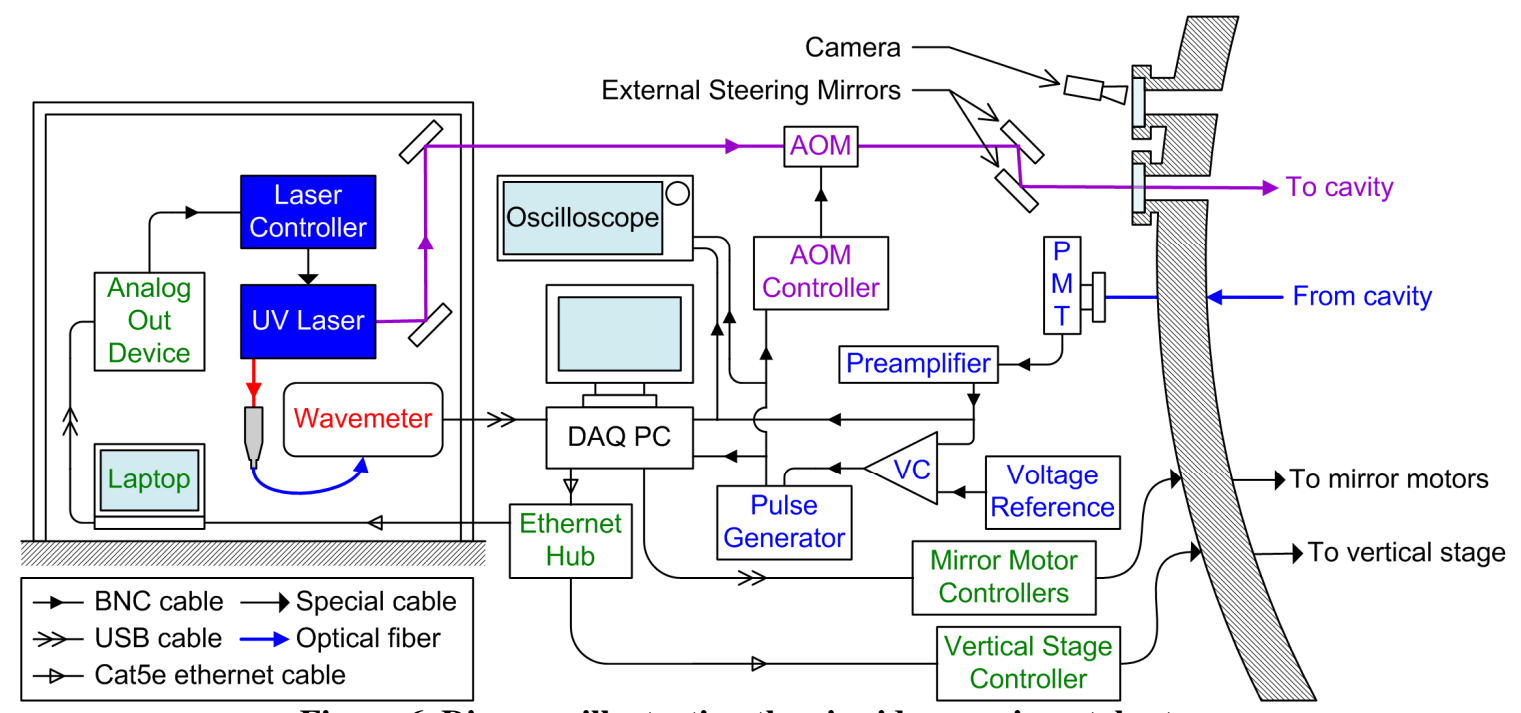

Figure 6. Diagram illustrating the air-side experimental setup.

8

American Institute of Aeronautics and Astronautics 
laser wavelength.

The UV laser light is directed by high reflectivity mirrors to a laser platform that sits next to the vacuum chamber. The UV beam passes through a Neos 35110-2-244-BR acousto-optical modulator (AOM), which is powered by a 31110-4DS AOM controller. Roughly $70 \%$ of the input beam power is coupled into the first order mode of the AOM output when the AOM is on. This first order beam propagates at a slightly different angle than the original laser beam that comes into the AOM. An iris is used to block off all of the light coming out of the AOM except the first-order beam. This first-order beam is then injected into the vacuum chamber via two external steering mirrors with fine-tuning knobs. The rough alignment of this UV beam is monitored using a video camera and a pair of irises built into the injection lens train of the physical cavity.

The output from the CRDS cavity is returned via optical fiber to a Hamamatsu R9110 photomultiplier (PMT) through a band-pass interference filter with a 10-nm bandwidth centered at $250 \mathrm{~nm}$. This PMT is powered by an Oriel 70705 PMT power supply. The output of the PMT is converted from current to voltage by a FEMTO DHPCA100 preamplifier operating at a gain of $10^{4}$ in low noise mode, which has a bandwidth of $14 \mathrm{MHz}$. Preparatory ringdown tests show that this is the highest gain setting that does not affect the value of the measured ring-down time constant (i.e. higher gain settings have smaller bandwidth, which starts to change the measured ring-down time).

The output from the preamplifier is fed to one of the channels on an AlazarTech ATS460 125-MHz DAQ card in the DAQ PC. The DAQ card was operated at only $100 \mathrm{MHz}$ as a precaution against over stressing the card. The preamplifier output is also fed to a LeCroy 314 oscilloscope for live monitoring and a custom voltage comparator circuit built into a Schmitt trigger configuration. The model of the voltage comparator chip is LM361 with a switching time of $20 \mathrm{~ns}$. It is powered by an Instek GPS-3303 laboratory power supply. Switching and stabilizing capacitors were added to improve the performance of the Schmitt trigger against random noise. The power lines between the power supply and the circuit were kept short for the same reason. The reference voltage was provided by a potentiometer-based voltage divider with a $9-\mathrm{V}$ battery as the voltage source. The non-inverting output of the circuit was used to complete the Schmitt trigger configuration while the inverting output was used to drive the triggering of a pulse generator.

The BK Precision 4030 pulse generator has an insertion delay of $60 \mathrm{~ns}$ (i.e. it takes $60 \mathrm{~ns}$ from the time when the triggering condition is met to when the pulse generation starts). The pulse width is set to $12 \mu \mathrm{s}$, which is more than ten times the typical ring-down time. Output of the pulse generator is fed into the AOM controller to initiate ringdown when the voltage comparator decides the signal in the cavity is high enough. This output signal is also fed into the DAQ PC to trigger the start of data acquisition and the oscilloscope for live monitoring. 


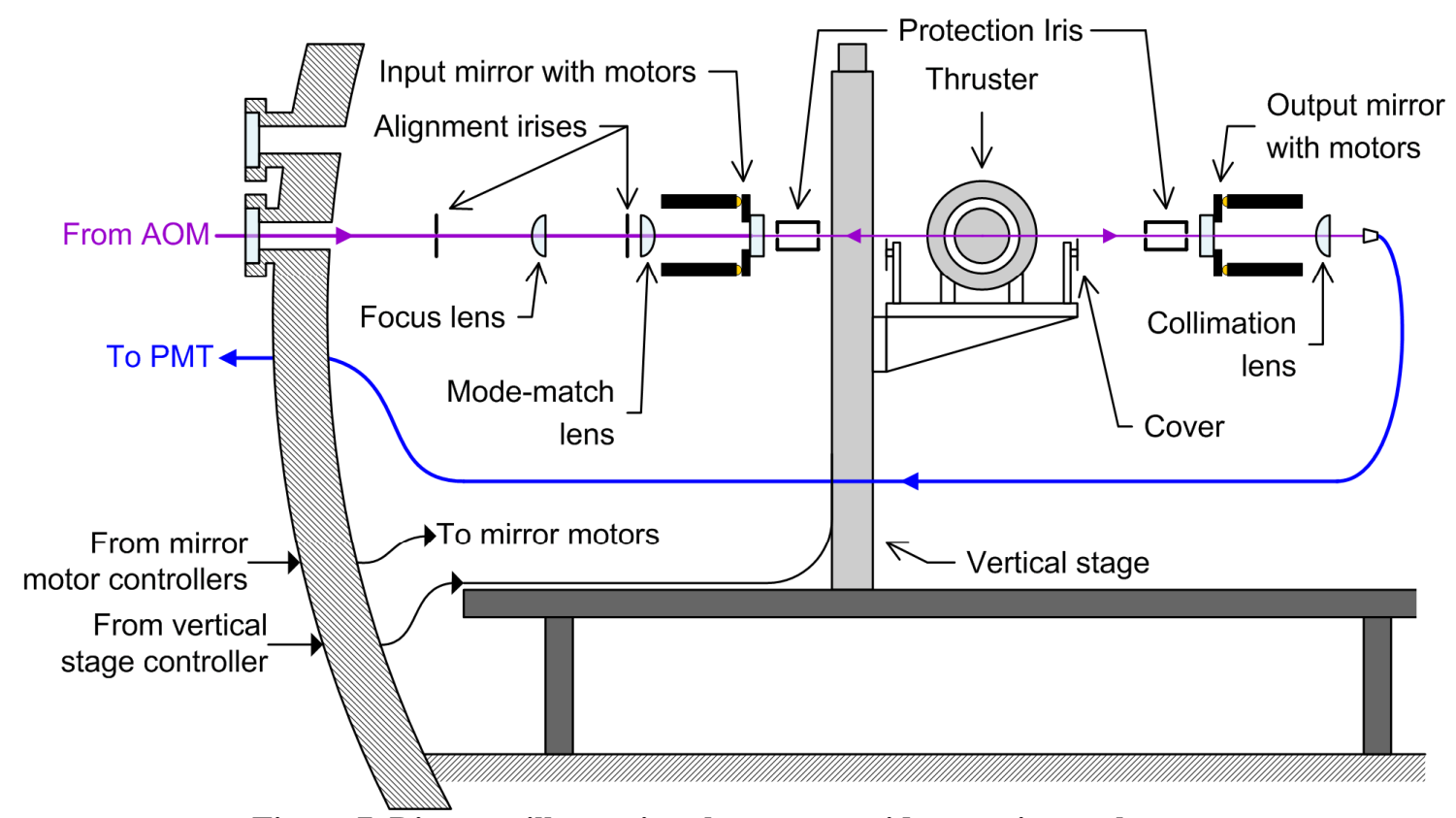

Figure 7. Diagram illustrating the vacuum-side experimental setup.

\section{Vacuum-Side Setup}

Figure 7 shows the vacuum-side experimental setup for the CRDS study. Figure 8 shows a composite photograph of the injection lens train being rough aligned. It is a composite photo because the camera cannot focus on the near and the far part of the lens train at the same time. There are no optics shown if Fig. 8. They are installed once the cavity has been properly positioned inside the vacuum chamber.

The first order beam from the output of the AOM is deflected by the external steering mirrors though a pair of alignment irises. The alignment irises are made of fluorescing discs that turn red when interacting with UV light. Precisely-drilled holes of $2 \pm 0.05 \mathrm{~mm}$ diameter are drilled into the center of each disc. The discs are mounted on two-axis translating mounts to help with the positioning of the alignment irises. The UV laser beam is usually slightly bigger than $2 \mathrm{~mm}$ in diameter by the time it reaches the first alignment iris. Parts of the beam beyond $2 \mathrm{~mm}$ fluoresce the iris and help with alignment. Fluorescence from the irises is monitored by an external camera through a viewport.

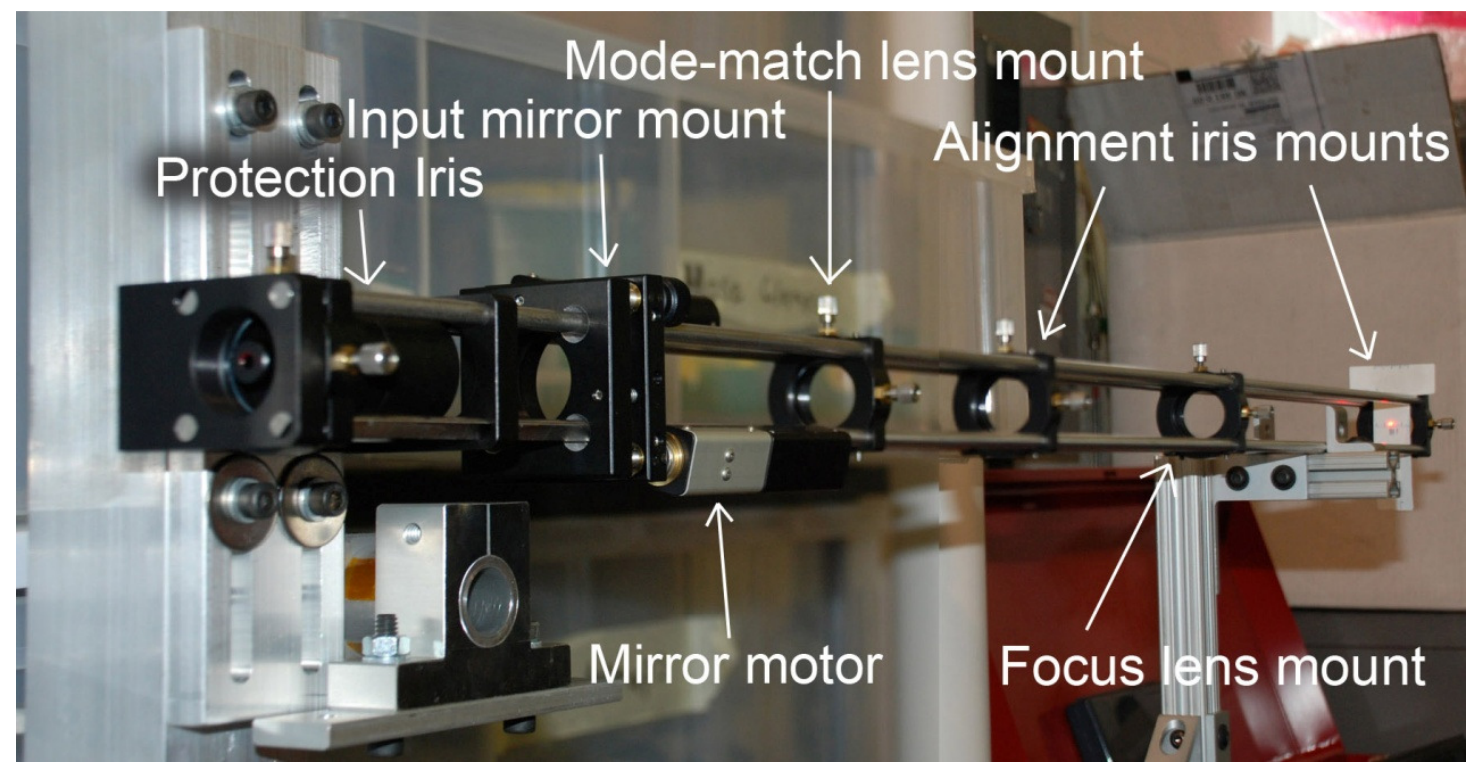

Figure 8. Photograph of the injection lens train being rough aligned.

10

American Institute of Aeronautics and Astronautics 
At this point, the beam going into the focusing lens is of known diameter and is approximately collimated. The focusing lens has a nominal focal length of $300 \mathrm{~mm}$ and is made of uncoated UV-grade fused silica. Its purpose is to bring the UV laser light down to a point of known diameter and location to aid in mode-matching.

The mode-matching lens, which comes next, matches the known position and diameter of the post-focusing-lens UV beam with the position and diameter of the $\mathrm{TEM}_{00}$ mode of the CRDS cavity. This lens has a nominal focal length of $125 \mathrm{~mm}$ and is made of uncoated UV-grade fused silica. Details on mode-matching can be found in work by Kogelnik and $\mathrm{Li}^{19}$

Next in the injection lens train is the input cavity mirror. The input and output cavity mirrors are custom fabricated by MLD Technologies. These mirrors are made from 1" diameter, 1/4" thick super-polished fused silica substrate. One side of each mirror is concave with a 1-m radius-of-curvature and coated for high reflectivity at $\sim 250$ $\mathrm{nm}$. The other side is flat and uncoated. The CRDS cavity beam resides at $4.0 \pm 0.5 \mathrm{~mm}$ downstream of the thruster channel exit plane and has a beam waist of $0.2-0.25 \mathrm{~mm}$, roughly matching the theoretical value calculated from equations in Kogelnik and Li's work. ${ }^{19}$ The mirrors sit on kinematic stages that have been modified to have motorized pitch and yaw axes. The input mirror mount is actuated by a pair of Thorlabs ZST6 micro stepper motors, while the output mirror mount is actuated by a pair of ZST13 motors.

There are also protection iris tubes between the Hall thruster plasma and each cavity mirror. These tubes have openings on each end that are $3.6 \mathrm{~mm}$ in diameter and are $\sim 80 \mathrm{~mm}$ long. The open space between the iris and the mirror mounts as well as along the rest of the lens train is sealed with transparent tape to protect against sputtered particles that are floating around the chamber during testing. In addition, there are two protection covers that extend axially downstream from the platform that holds the Hall thruster. Since we are assuming the thruster is axisymmetric, CRDS results can be obtained by scanning only half of the thruster in the vertical direction. When the CRDS sensor is not in use, the entire thruster platform can be raised so that the protective covers sit right over the holes of the two protection iris tubes. Although not shown this way in Fig. 7, the covers sit at only $5 \mathrm{~mm}$ in front of their respective protection iris openings when in place. These covers also act as an additional position reference since we will know a priori the P-position at which the covers cut off the cavity beam. All materials that sit near the thruster are made of non-magnetic material and do not perturb the thruster's magnetic field.

Vertical position of the thruster platform is controlled by an Aerotech ATS62150 motion stage with a travel of $1.5 \mathrm{~m}$ and is powered by a VS-U33 stepper motor. The resolution of this motion stage is $0.01 \mathrm{~mm}$. The accuracy of this stage is $\pm 0.1 \mathrm{~mm}$, determined by how readily the cutoff of the UV beam by the protection covers can be detected. The position of this vertical stage is monitored by an HS25 1024-line relative encoder from BEI Sensors. The thruster sits on an adjustable platform the lower half of which is supported by the vertical stage. The tilt of this platform is adjusted so that the channel exit plane of the thruster is parallel to the travel axis of the vertical stage to within $\sim 1^{\circ}$.

The light that comes out of the output cavity mirror is collected by the collimation lens and focused into the collection optical fiber. The collimation lens has a nominal focal length of $50 \mathrm{~mm}$ and is made of uncoated UVgrade fused silica. The collection optical fiber has a 550- $\mu \mathrm{m}$ core diameter, is $9 \mathrm{~m}$ in length, and has a total attenuation of $\sim 25 \%$ at $250 \mathrm{~nm}$. This fiber was in use for a total of about 40-50 hours while showing no noticeable signs of degradation. Output from this optical fiber is fed into the PMT on the air-side for signal collection.

With the optical parts of the CRDS cavity described, we now turn to the structure that holds the optics in place. Figure 9 shows a photograph of the actual vacuum-side experimental setup. The physical cavity is made of four aluminum members welded together. Each member has a $2 " \times 2$ " hollow square cross section. The walls of the members are $1 / 4$ " thick. This design provides good rigidity while meeting thermal driven criterion. The two legs used to support the physical cavity are made of carbon fiber rods connected by custom aluminum connections. Each leg is supported by three urethane vibration isolators. In Fig. 9, the protective covers are sitting right in front of their respective protection iris tube openings. The transparent tape that protects the lens train from sputtered particles is somewhat visible in this photograph. 


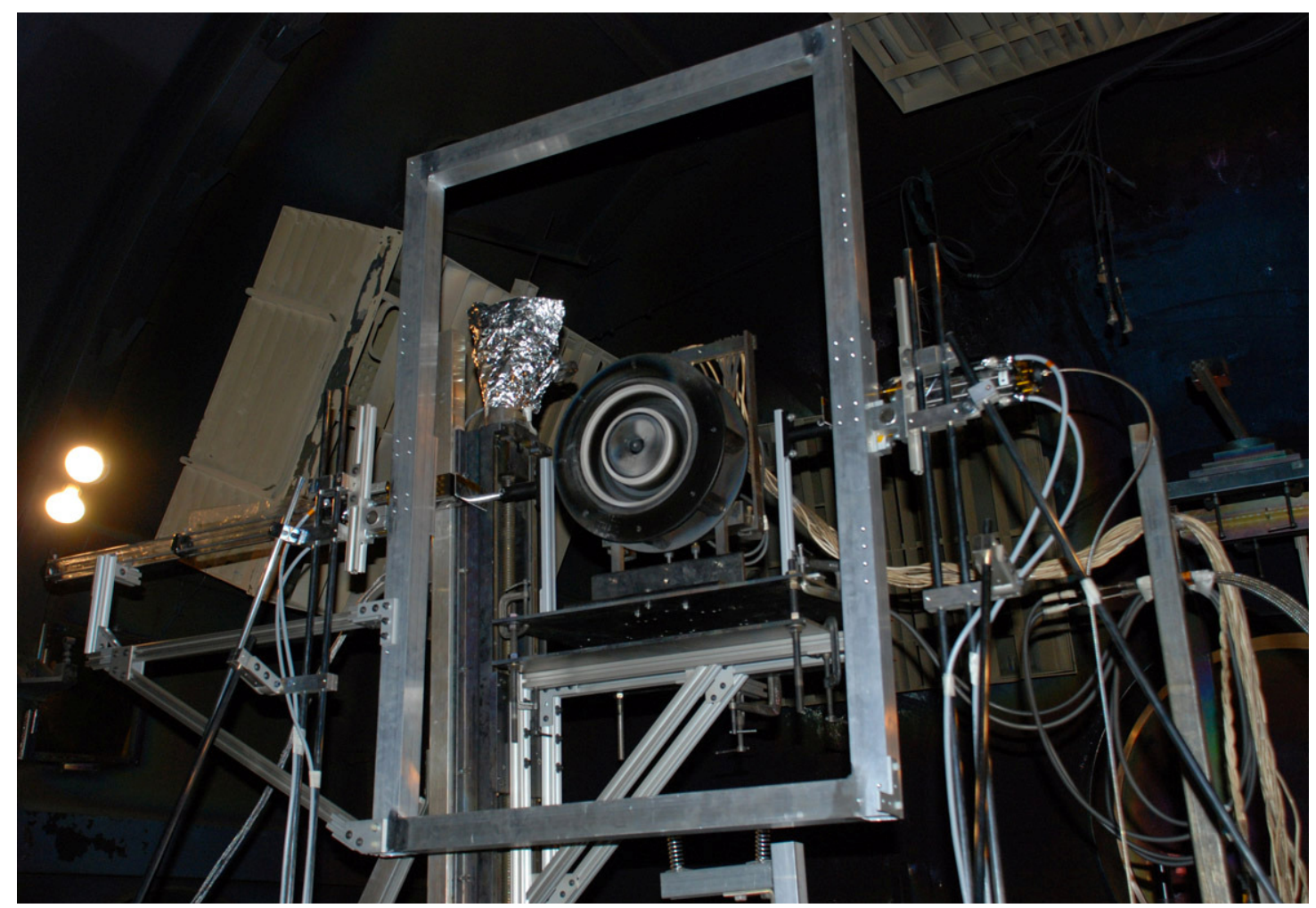

Figure 9. Photograph of the vacuum-side experimental setup.

\section{E. Curve-Fit Algorithm for the Ring-Downs}

Curve-fitting the raw data for the ring-down time constant is accomplished by using an exponential fit subroutine in Labview v9. This sub-routine uses the bisquare weights method to perform a curve-fit of the data to Eq. (15). The bisquare method is good at rejecting outliers, which may show up as a result of noise spikes.

$$
\mathrm{S}=\mathrm{S}_{0} \exp \left(\mathrm{C}_{1} \mathrm{t}\right)+\mathrm{C}_{2}
$$

where $\mathrm{S}$ is the signal intensity, $\mathrm{S}_{0}$ is the signal amplitude, $\mathrm{C}_{1}$ is inverse of the decay time constant, and $\mathrm{C}_{2}$ is an offset constant. Only negative values of $\mathrm{C}_{1}$ are allowed in the curve-fit so the raw output for time constant is always negative. Lehmann, Berden, and Engeln ${ }^{23}$ suggest using ten times the time constant worth of data when there is a possibility of the offset constant varying from trace to trace. This is the driving factor for setting the pulse width to $12 \mu \mathrm{s}$ (highest ring-down time is $1 \mu \mathrm{s}$ ). All data were taken at $100 \mathrm{MHz}$. The number of data points that comprise each ring-down varies depending on how many points are needed to get approximately 10 times the time constant worth of data. The number of points changes because the ring-down time constant decreases as the mirrors degrade during a test. Also, to ensure that the ring-down has actually begun at the start of data recording, there is a time delay between when the DAQ PC receives the AOM trigger and when it starts recording data. A time delay of 0.55 $\mu$ s was experimentally found to guarantee that the data recording starts after the ring-down has begun. 
Figure 10 shows an example of a single ring-down with the associated curve-fit performed by the DAQ PC. This particular trace was obtained prior to the pump-down of the vacuum chamber for the first round of CRDS testing. The data in this figure is representative of data obtained at the start of each round of CRDS testing.

During an actual test, the custom Labview program that controls the data acquisition only records the time constant extracted from each scan. From the 100-200 time constants recorded this way per wavelength, statistical calculations are carried out on-the-fly and displayed. The alignment of the cavity can in part be judged by the average of and the spread in the ring-down time. Benchmark testing of the Labview program showed that it is capable of curve-fitting up to $\sim 700$ traces per second and is not a bottleneck for data acquisition.

\section{Data Reduction}

\section{A. Computing the Line-Integrated and Absolute Boron Density}

To calculate the line-integrated boron density, one needs to know the empty cavity ring-down time. Normally, this value remains constant throughout a test, but the degradation of mirrors in a Hall thruster environment is rapid enough that the value is not a constant. To calculate the empty cavity ring-down time it is assumed that the degradation is due to accumulation of some kind of material on the mirrors' reflective surfaces. This material is treated as an added absorber that grows linearly with time for a given experimental geometry. The exact growth rate may vary with geometry, but is a constant for a given geometry. Mathematically, this constant rate deposition is the same as adding a growth rate times time term into the bracket of Eq. (2). The empty cavity ring-down can the be modeled by Eq. (16),

$$
\tau_{0}=\frac{1}{\mathrm{at}+\mathrm{b}}
$$

where $\mathrm{a}$ is a constant that represents the growth rate of the deposition and $\mathrm{b}$ is a constant that correlates with the mirror reflectivity at the start of the scan. Data points on the edges of the scan range are assumed to be out of resonance and are used to perform the curve-fit for the constants a and b. (Also see Section IV.D) The data points inbetween are assumed to be a part of the $249.848 \mathrm{~nm}$ transition lineshape and integrated to find the total line-integrated density. The exact range of integration is the same for all CRDS data analyzed and is $12.4 \mathrm{pm}$ wide centered at 249.848 $\mathrm{nm}$ based on the wavemeter measurements. The location of the $249.848 \mathrm{~nm}$ marker from the wavemeter measurements corresponds well with the location of peak signal for all obtained data.

Figure 11 shows an example of how the curve-fitting to find the empty cavity ring-down time works. This particular trace comes from $300 \mathrm{~V}, 20 \mathrm{mg} / \mathrm{s}$ operating at a cavity position of $\mathrm{P}=0.5$. As the figure shows, the curve-fit is pretty much shaped like a line due to the small change in ring-down time relative to the amplitude of the ring-down time. Additional data analysis shows that using a linear fit also works fine.

As a side note, the mirrors were cleaned between each round of CRDS testing (there were three rounds with 2-3 conditions tested per round), and each time the mirror

13 ring-down time is found.

Black dots are data points, red circles near the two edges are data points used to perform curve-fit, and blue line is the resulting fit.
Figure 11. An example of how the empty cavity

American Institute of Aeronautics and Astronautics 


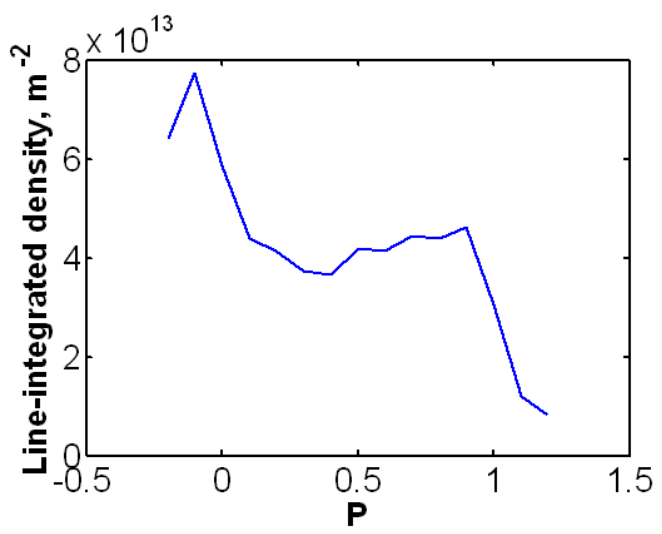

Figure 12. Example plot of line-integrated boron density as a function of non-dimensionalized vertical position.

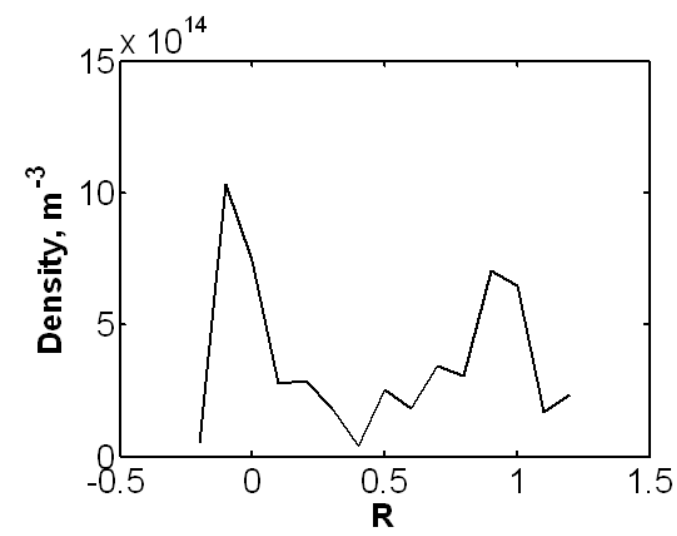

Figure 13. Example plot of boron density as a function of non-dimensionalized radial position.

reflectivity recovers to its optimum value. Further details on mirror cleaning will be discussed in a future paper.

Once the empty cavity ring-down time is determined, we can use Eq. (3) to calculate the absorbance and Eq. (4) to calculate the line-integrated boron density of the specific ground state we are measuring. We can then use the onion-peeling method to convert the line-integrated density as a function of the vertical coordinate, $\mathrm{p}$, to absolute density as a function of the radial coordinate, $r$.

Figure 12 shows the line-integrated boron density measured for the 6-kW Hall thruster operating at $300 \mathrm{~V}, 30$ $\mathrm{mg} / \mathrm{s}$. Figure 13 shows the same data after the onion-peeling method is applied. As with the correspondence between $\mathrm{r}$ and $\mathrm{p}$, the associated non-dimensional form $\mathrm{R}$ and $\mathrm{P}$ also have a one-to-one relationship. Each concentric onion ring has a width of 0.1 with the corresponding $\mathrm{R}$ value being halfway between the two radii that bound each ring.

\section{B. Boron Velocity Modeling}

To calculate the boron flux, information about the axial velocity of the boron at each measured location is needed. For the purpose of this paper, a very simple model, combined with Sigmund-Thompson distribution data measured by Tao and Yalin ${ }^{22}$ will be used to approximate the boron axial velocity.

The model is a simple two point-source model and is formulated in the R-Z plane. Sputtered particles are assumed to radiate isotropically outward in a threedimensional fashion from two points in the R-Z plane (or two rings in 3D space) at a fixed speed. The speed used is the $23 \mathrm{~km} / \mathrm{s}$ calculated as previously described using data obtained by Tao and Yalin. ${ }^{22}$ Figure 14 illustrates the key concepts behind this model. Particles that arrive at the interrogated location from each point source carry an axial

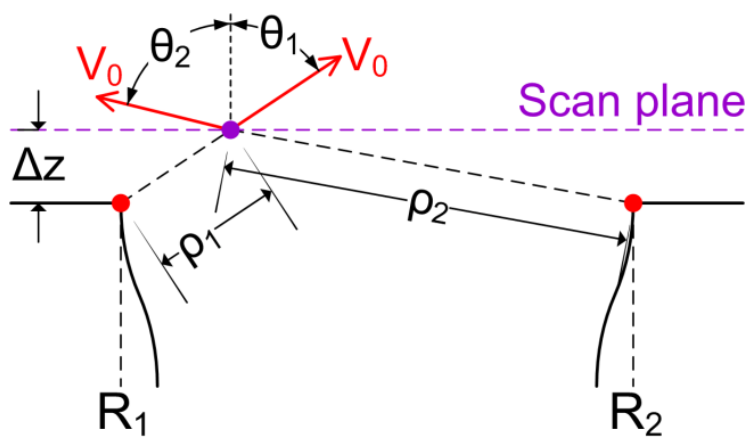

Figure 14. Illustration for the two point-source boron velocity model.

velocity that can be calculated from Eq. (17), which is derived from simple geometry,

$$
\mathrm{V}_{\mathrm{Z}, \mathrm{i}}=\mathrm{V}_{0} \frac{\Delta \mathrm{z}}{\sqrt{\left(\mathrm{r}-\mathrm{R}_{\mathrm{i}}\right)^{2}+(\Delta \mathrm{z})^{2}}}
$$

where $\mathrm{V}_{0}$ is the speed of the sputtered particles, $\Delta \mathrm{z}$ is the distance from the channel exit plane to the CRDS laser scan plane, and is $4 \pm 0.5 \mathrm{~mm}, r$ is the radial location of the interrogated point, and $R_{i}$ is the radial location of the i-th source ( $i=1$ or 2 ). Since particles are radiating out in 3D space, the particle density scales as inverse of the square of the distance. The fractional contribution by each source can be computed from the distance between the interrogated point and each source using Eq. (18), 


$$
f_{i}=\frac{\rho_{i}^{-2}}{\rho_{1}^{-2}+\rho_{2}^{-2}}
$$

where $\rho_{\mathrm{i}}$ is the distance from the $\mathrm{i}$-th source $(\mathrm{i}=1$ or 2$)$ to the interrogated location. The average axial velocity of the sputtered boron particles at the interrogated point can then be calculated using Eq. (19).

$$
\mathrm{V}_{\mathrm{Z}}=\mathrm{f}_{1} \mathrm{~V}_{\mathrm{Z}, 1}+\mathrm{f}_{2} \mathrm{~V}_{\mathrm{Z}, 2}
$$

The boron flux can now be calculated by combining the density data with the modeled axial velocity. Then, the area of each onion ring can be calculated from simple geometry. Lastly, the total rate of boron sputtering, for the ground state measured, can be derived.

An important assumption in the above simple boron velocity model is that the sources are points in the R-Z plane. In reality, there is contribution to the particles at the interrogated point from neighboring azimuthal locations. The axial velocity of the particles from these locations will be lower than the axial velocity calculated in the model because the arrival angle, $\theta_{\mathrm{i}}$, is always greater when the particles come from out-of-plane. Thus, the calculated velocity is a rough estimate of the axial velocity upper bound.

Additionally, the sputtered boron generally do not radiate isotropically. However, incorporating the anisotropy will not change the answer much because the outer-wall-source contributes very little to the axial velocity near the inner wall, and vice versa. For example, at $\mathrm{R}=0$, the contribution from the outer-wall-source is $2 \%$, while at $\mathrm{R}=$ 0.1 , it is $5 \%$. As will be seen in the plotted results later, the majority of the sputtered boron is located very near their respective walls and contribution from the other wall can be approximated as a higher order effect.

In theory, the lineshape obtained in the CRDS data can be used to check some of the assumptions made in the velocity modeling since the CRDS laser beam is affected by Doppler shift. This is left for future work.

\section{Correlating Boron Flux to Sputter Rate}

The conversion from specific-ground-state boron flux to sputter rate can be performed with Eq. (20),

$$
\mathrm{SR}=\rho_{\mathrm{BN}}{ }^{-1} \mathrm{~m}_{\mathrm{BN}}\left(\frac{\mathrm{g}_{1 / 2}+\mathrm{g}_{3 / 2}}{\mathrm{~g}_{3 / 2}}\right) \Phi_{\mathrm{B}, 3 / 2}
$$

where SR is the sputter rate in $\mathrm{m}^{3} / \mathrm{s}, \rho_{\mathrm{BN}}$ is the mass density of the $\mathrm{BN}$ that the channel walls are made from in $\mathrm{kg} / \mathrm{m}^{3}, \mathrm{~m}_{\mathrm{BN}}$ is the mass of a single BN molecule in $\mathrm{kg}, \mathrm{g}_{1 / 2}$ is the degeneracy of the $2 \mathrm{p}[1 / 2]_{2}$ ground state of boron, $\mathrm{g}_{3 / 2}$ is the degeneracy of the $2 \mathrm{p}[3 / 2]_{4}$ ground state of boron, and $\Phi_{\mathrm{B}, 3 / 2}$ is the flux of boron in the $2 \mathrm{p}[3 / 2]_{4}$ ground state in $\mathrm{s}^{-1}$. The mass density of BN is given by the manufacturer as $2.0 \mathrm{~g} / \mathrm{cm}^{3}$. The above equation is applicable when the following assumptions apply,

1. All sputtered boron are in the ground states. ${ }^{24}$ This means they are not part of a BN molecular complex, ${ }^{25}$ in an excited state, or in a charged state.

2. A Boltzmann distribution can be used to characterize the population of the fine structure levels within the ground state multiplet. Past research has shown the aforementioned assumption to be a good approximation even though the sputtered particles are not generally at thermal equilibrium. ${ }^{24}$ This assumption means that the ratio of total ground state population to the $2 \mathrm{p}[3 / 2]_{4}$ ground state (lower state of the $249.848 \mathrm{~nm}$ transition) is given by ratio of the total degeneracy to the degeneracy of the $2 \mathrm{p}[3 / 2]_{4}$ state. The value of this ratio is 1.5 because the degeneracy is 2 and 4 for the $2 \mathrm{p}[1 / 2]_{2}$ and $2 \mathrm{p}[3 / 2]_{4}$ states, respectively.

3. Boron and nitrogen are sputtered in a one-to-one ratio, which is true at steady state according to known sputtering physics and there has been no evidence to the contrary. For every ground-state boron detected, the total mass leaving the thruster is equal to the mass of one boron atom plus one nitrogen atom.

\section{Uncertainty and Sources of Error}

The uncertainty in the extracted boron density comes from spread in the average ring-down time. One can use Eq. (9) to compute the minimum detectable absorbance for each data trace. Assuming the signal is roughly triangular in nature (i.e. that it has a single peak) one can calculate the signal-to-noise ratio of a data trace. The signal-to-noise ratio (SNR) is defined as the magnitude of the peak signal divided by the root-mean-square of the noise. The relative uncertainty in the line-integrated density corresponding to the minimum detectable absorbance is 
just the inverse of the SNR. Since the uncertainty in the ring-down time is computed with a $95 \%$ confidence interval, the relative uncertainty in the line-integrated density derived as previously described also comes with a $95 \%$ confidence interval. Further statistical analysis can then be carried out using the nature of the onion-peeling method to compute the uncertainty in each boron density data point. To simplify the analysis, an average relative uncertainty for line-integrated density is computed for each operating condition. The relative uncertainty in the boron density is the same by standard error propagation.

The uncertainty in the relative sputter rate should be dominated only by the uncertainty in the boron density measurement. Uncertainty in the velocity modeling should have only a higher order effect on the relative sputter rate as long as it is consistent for all operating conditions. This is because the experimental data from work by Tao and Yalin ${ }^{22}$ show that the sputtered velocity is not strongly dependent on the energy of the bombarding particle and the LIF data from a previous work ${ }^{26}$ show that the variation in the average angle of incidence near the exit plane for different operating conditions is small $\left(\sim 10^{\circ}\right.$ spread for 300 and $600 \mathrm{~V}$ operation). With that said, it is possible that the axial velocity of the sputtered boron is sensitive to even such a small spread in bombardment angle and will require comparison to control experiments and finite-element analysis to check. This is left for future work. For the purpose of this paper it is assumed that the uncertainty in the relative sputter rate caused by variation in the axial velocity of the sputtered boron due to differences in operating conditions is negligible compared to the uncertainty propagated from the boron density measurement.

The uncertainty in the absolute sputter rate, on the other hand, is strongly influenced by the velocity modeling. The computed average speed of the Sigmund-Thompson distribution is strongly sensitive to the fitting parameters. For instance, a change in the value of $\mathrm{m}$ by 0.1 can cause a $10 \%$ change in the computed average speed. Similarly, the exact boundary of integration has a strong influence on the value of the computed average speed. Indeed, the best approach to obtaining the axial boron velocity is most likely to measure it directly with boron LIF, but boron LIF in a Hall thruster environment is a completely untested diagnostic. For the purpose of this paper, 50\% is assigned as the contribution of uncertainty in the absolute sputter rate due to the simplicity of the velocity model used. Refinement of the velocity model through kinetic simulations should greatly reduce this uncertainty, as would boron LIF velocimetry experiment. The CRDS lineshape can further be predicted by the use of a refined velocity model for comparison to measured CRDS lineshape. This comparison could potentially give a more accurate estimate of the uncertainty. These activities are left for future work.

Another source of error contributing to the uncertainty in the absolute sputter rate is the possibility of undercapturing the full lineshape in certain high SNR scans. Some lineshapes do not appear to have completely flattened out by the edge of the scan range. This is often difficult to discern because the lineshapes seem to terminate pretty close to the edges of the scan range. The short scan range is an unfortunate artifact of using direct beam injection to deliver the UV laser light. The slight change in beam angle at the UV laser output leads to variation in the modematch at the CRDS cavity. If too large of a scan range is used, at the edges of the scan range, the laser light will couple to higher-order modes. Note the laser mode-hop-free range was not a limiting factor in the scan range. While it is unclear if all of the $249.848 \mathrm{~nm}$ lineshape is captured from these select scans, it is assumed that the majority is captured since other scans show proper flattening out at the extreme ends. Under-capturing the lineshape will lead to under-estimate of the sputter rate, but this under-estimation should be systematic because all of the lineshapes appear to be roughly triangular with similar widths. The similarity in the lineshape is not surprising since the lineshape is driven by similar geometries and the same Sigmund-Thompson-VDF-based Doppler broadening. The maximum systematic error is tentatively listed as $20 \%$.

Other sources of error contributing to the uncertainty in the absolute sputter rate include uncertainty in the mass density of the BN and uncertainty in the correlation ratio between the total sputtered boron density and the boron density of the $2 \mathrm{p}[3 / 2]_{4}$ ground state. The former uncertainty is expected to be a few percent at most, which is negligible compared to the potential error in the axial velocity. The latter uncertainty depends on how much of the boron is in the excited states, the charged states, molecular complex, or the $2 \mathrm{p}[1 / 2]_{2}$ ground state. No quantitative data about the amount of sputtered particles that are ejected as molecular complex is currently published, though past QCM sputtering studies, including X-ray photoelectron spectroscopy (XPS) measurements, indicate that sputtered boron is primarily in atomic form. ${ }^{27}$ The ratio of $2 \mathrm{p}[1 / 2]_{2}$ to $2 \mathrm{p}[3 / 2]_{4}$ ground states is assumed to be calculable by assuming thermal equilibrium distribution of states. Confirmation is left for future works. A rough analysis of the fraction of excited and ionized state boron to the total boron population was performed using the cross section data collected by Kim and Stone ${ }^{28}$ combined with plasma density measurement inside the 6-kW collected by Reid. ${ }^{29}$ The main results were that negligible amount of excited and ionized boron came off of the channel walls from xenon bombardment, but a non-negligible amount of boron ions may be formed as boron neutrals travel through the channel before reaching the cavity laser beam. The fraction of ionized boron at the CRDS 
measurement plane was estimated to have a systematic error of $20 \%$. That is to say, the absolute sputter rate can be underestimated by up to $20 \%$.

\section{Results}

\section{A. Density Plots}

Figure 15 shows sample raw CRDS traces for the $300 \mathrm{~V}, 30 \mathrm{mg} / \mathrm{s}$ operating condition. This data set has the lowest uncertainty of all of the data set and nicely illustrates the kind of signal to expect when the CRDS diagnostics is working properly. In the figure, $\mathrm{P}$ is the non-dimensionalized vertical coordinate. In contrast, Fig. 16 shows sample raw CRDS traces for the $150 \mathrm{~V}, 30 \mathrm{mg} / \mathrm{s}$ condition, where the measured sputter rate was about the same as the minimum detectable sputter rate. While sputtering information can still be extracted for this condition once the data is put through the data reduction methods previous described, the extracted sputter rate is close to the detection threshold. The offsets between traces shown in Figs. 15 and 16 are a result of continuous degradation in the mirror reflectivity as the test proceeded.

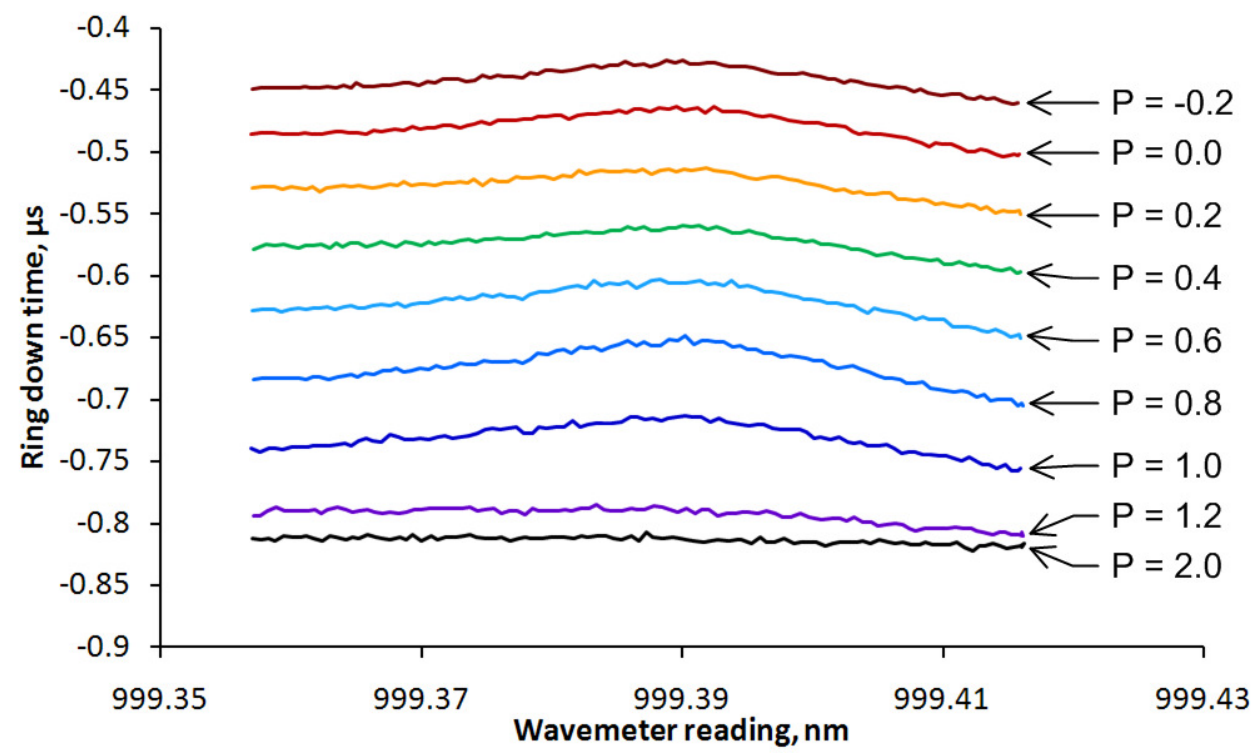

Figure 15. Sample raw CRDS traces for the 6-kW Hall thruster operating at $300 \mathrm{~V}, 30 \mathrm{mg} / \mathrm{s}$

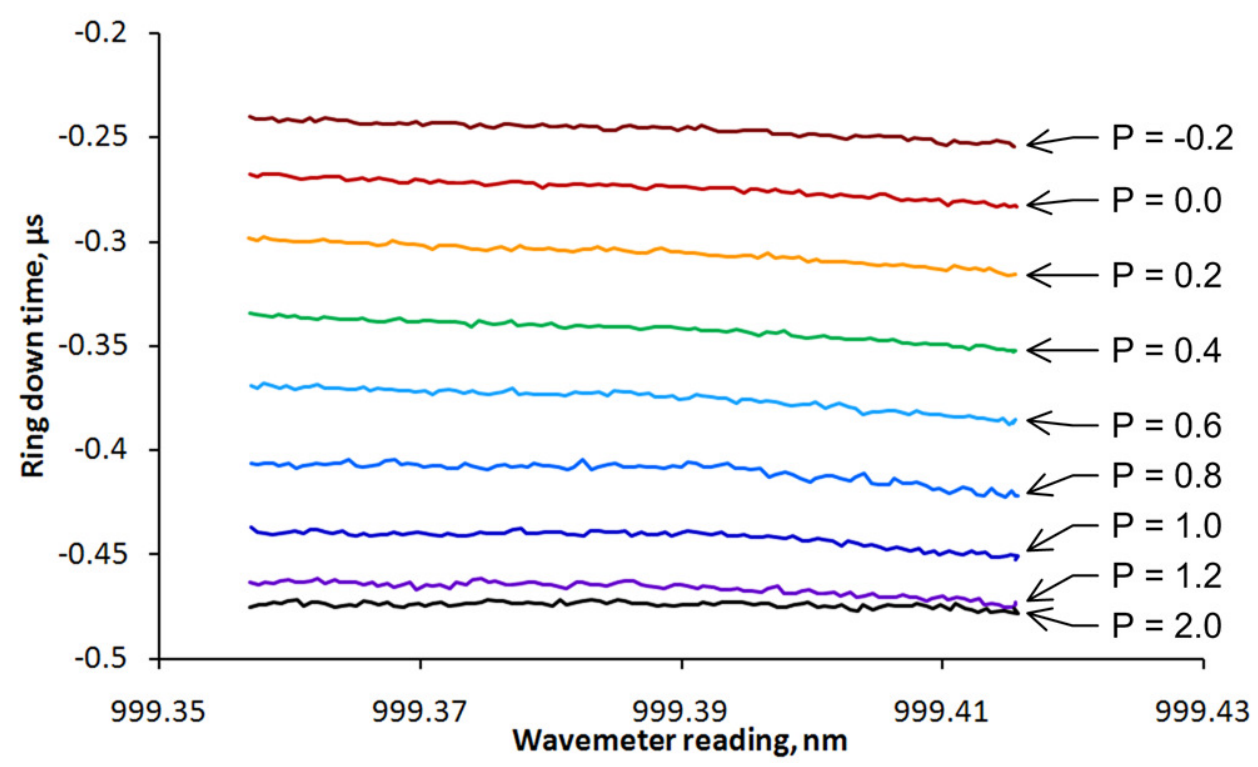

Figure 16. Sample raw CRDS traces for the 6-kW Hall thruster operating at $150 \mathrm{~V}, 30 \mathrm{mg} / \mathrm{s}$. 17

American Institute of Aeronautics and Astronautics 


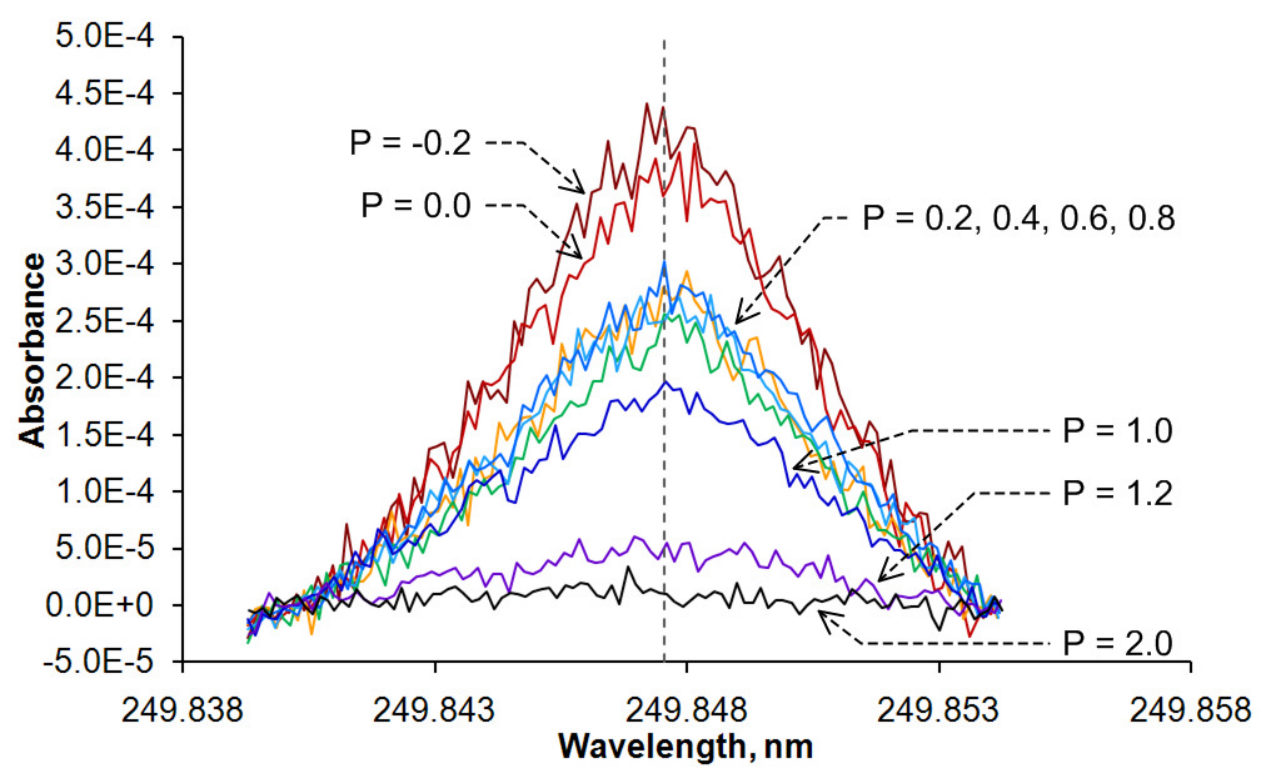

Figure 17. Sample processed CRDS traces for the 6-kW Hall thruster operating at $300 \mathrm{~V}, 30 \mathrm{mg} / \mathrm{s}$.

Figure 17 shows the $300 \mathrm{~V}, 30 \mathrm{mg} / \mathrm{s}$ data set after it is processed. The amplitude and shape of the signal as well as the amount of noise in the data is much easier to see in this figure than in Fig. 15. Figure 17 also shows a vertical dashed line indicating the average wavelength of the $249.848 \mathrm{~nm}$ transition as reported by the NIST atomic spectra database, ver. 4 (last updated September 2010). The exact value is $249.84755 \mathrm{~nm}$, which is the reason why the line does not line up with the $249.848 \mathrm{~nm}$ marker on the wavelength axis. The lineshape shows good symmetry because the broadening is driven by Doppler effect and roughly half of the UV photons in the cavity are traveling in each direction at a time. In general, the raw data recorded in this experiment show shapes that look Gaussian but could just as easily be described by a triangle for the amount of noise present. However, the true lineshape should not be Gaussian because the distribution function is not Maxwell-Boltzmann. ${ }^{30}$ It is currently unclear why the shapes of the distribution functions do not match theoretical prediction in part because the velocity model is still being refined. However, since the density is calculated by integrating over the entire lineshape, this discrepancy in lineshape does not affect the uncertainty in the result.

Figures 18 to 24 show the line-integrated density as a function of vertical position and the corresponding boron density as a function of radial position for the $6-\mathrm{kW}$ Hall thruster at various operating conditions. In each figure, the left sub-plot shows the line-integrated density data and the right sub-plot shows the absolute density results calculated from the onion-peeling method.
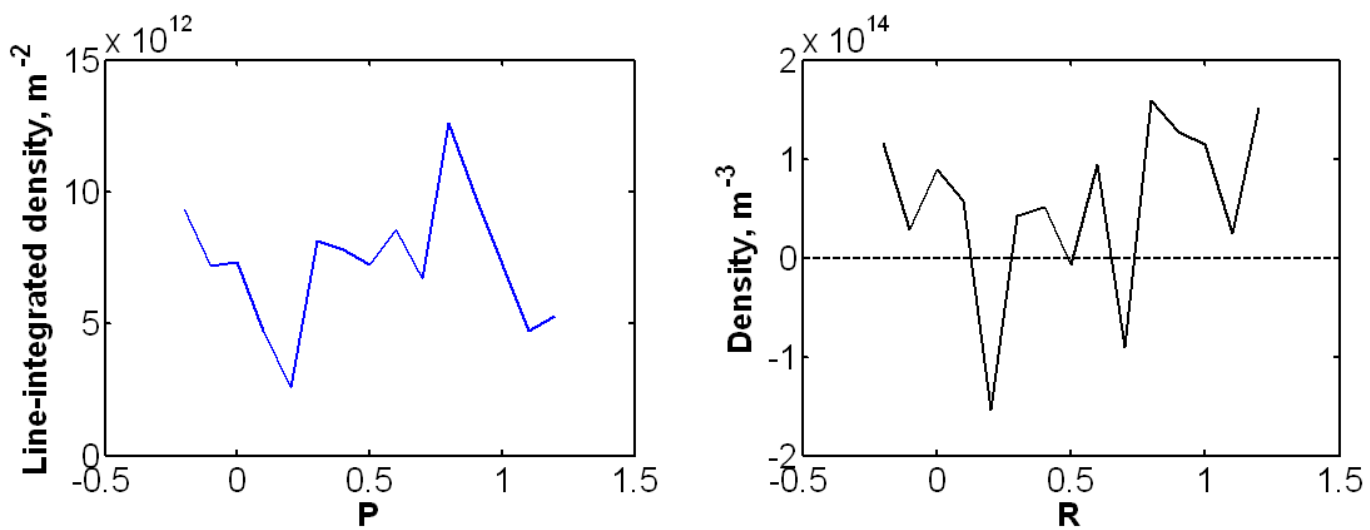

Figure 18. The line-integrated density plot and the corresponding boron density plot for the 6-kW Hall thruster operating at $150 \mathrm{~V}, 10 \mathrm{mg} / \mathrm{s}$. 

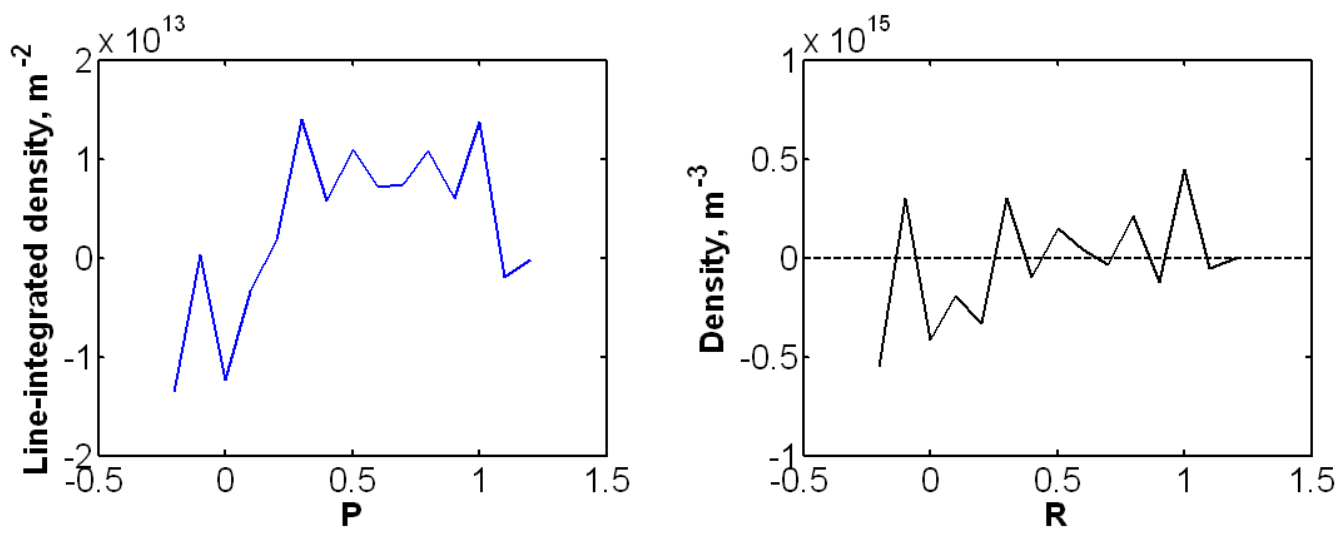

Figure 19. The line-integrated density plot and the corresponding boron density plot for the 6-kW Hall thruster operating at $150 \mathrm{~V}, 20 \mathrm{mg} / \mathrm{s}$.
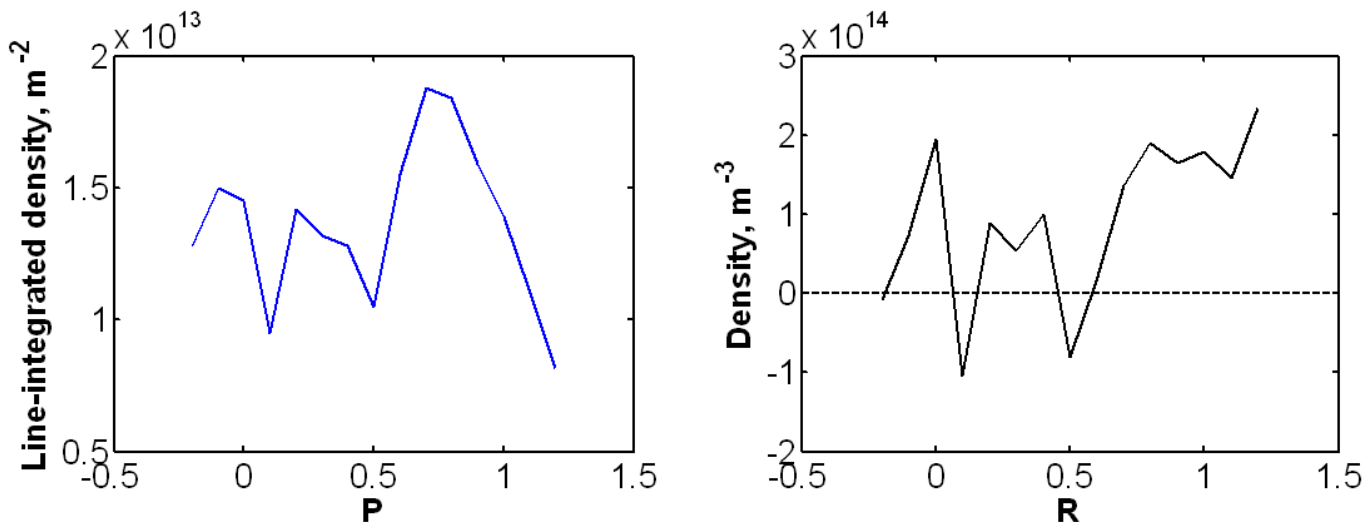

Figure 20. The line-integrated density plot and the corresponding boron density plot for the 6-kW Hall thruster operating at $150 \mathrm{~V}, 30 \mathrm{mg} / \mathrm{s}$.
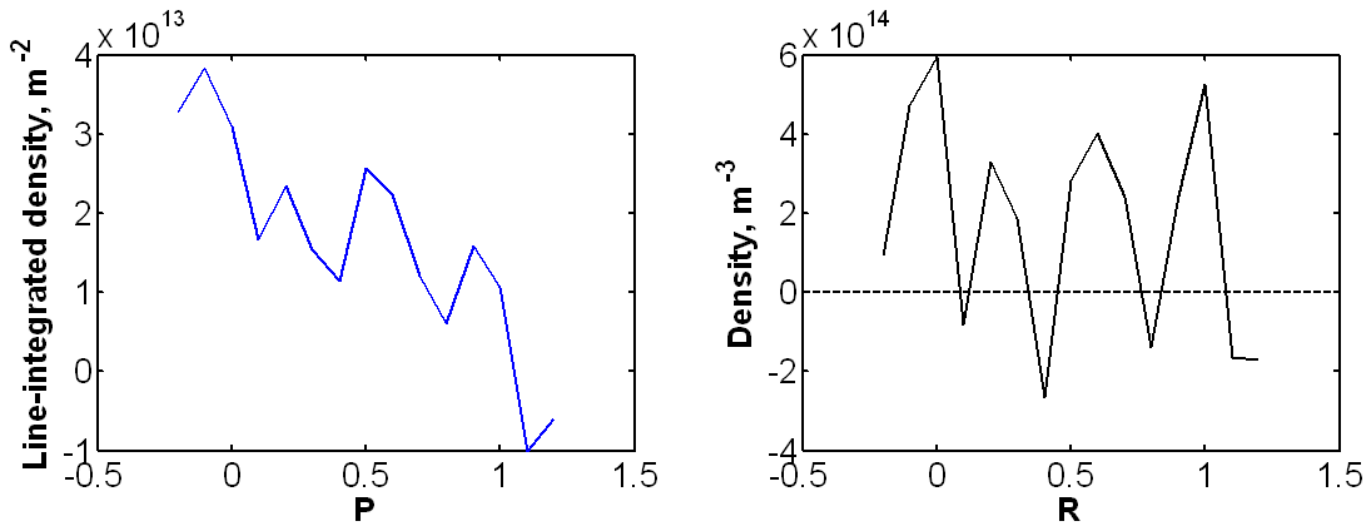

Figure 21. The line-integrated density plot and the corresponding boron density plot for the 6-kW Hall thruster operating at $300 \mathrm{~V}, 10 \mathrm{mg} / \mathrm{s}$. 

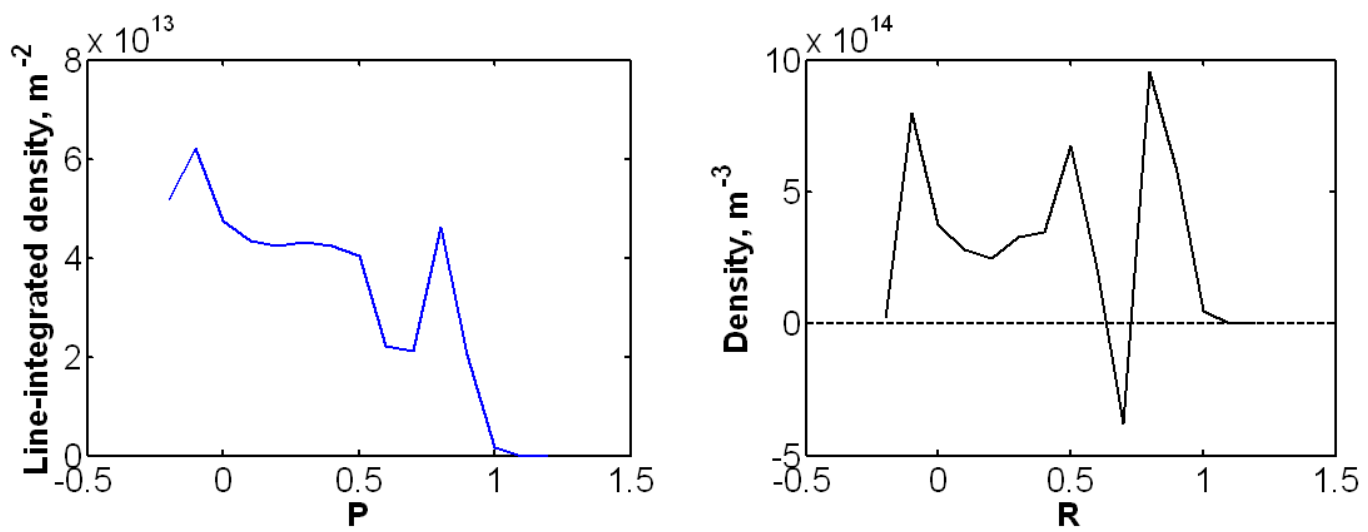

Figure 22. The line-integrated density plot and the corresponding boron density plot for the 6-kW Hall thruster operating at $300 \mathrm{~V}, 20 \mathrm{mg} / \mathrm{s}$.
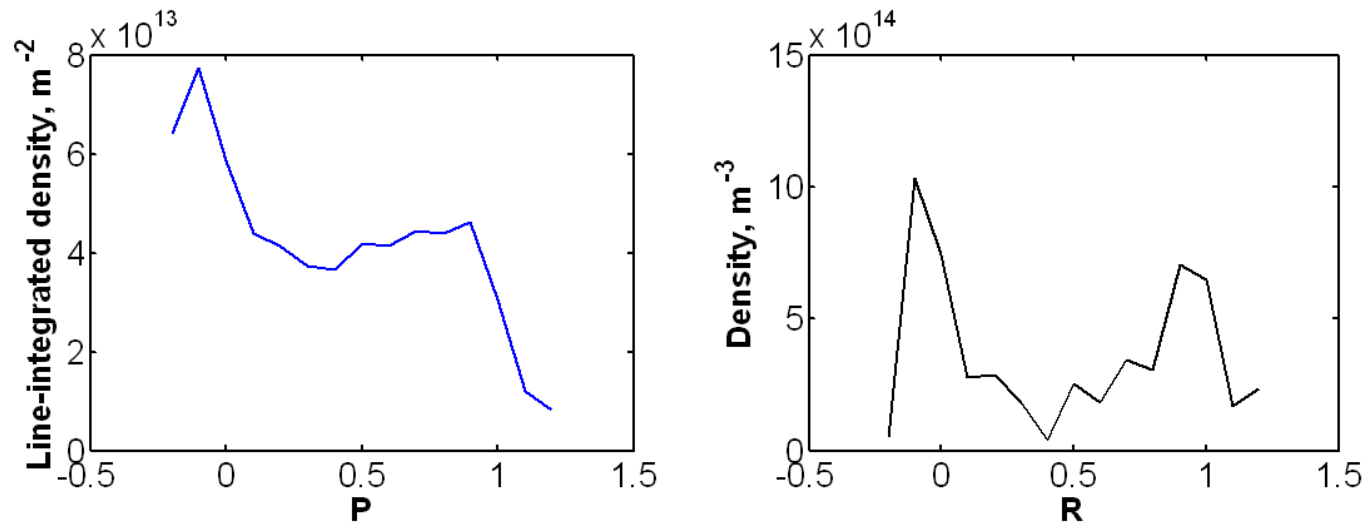

Figure 23. The line-integrated density plot and the corresponding boron density plot for the 6-kW Hall thruster operating at $300 \mathrm{~V}, 30 \mathrm{mg} / \mathrm{s}$.
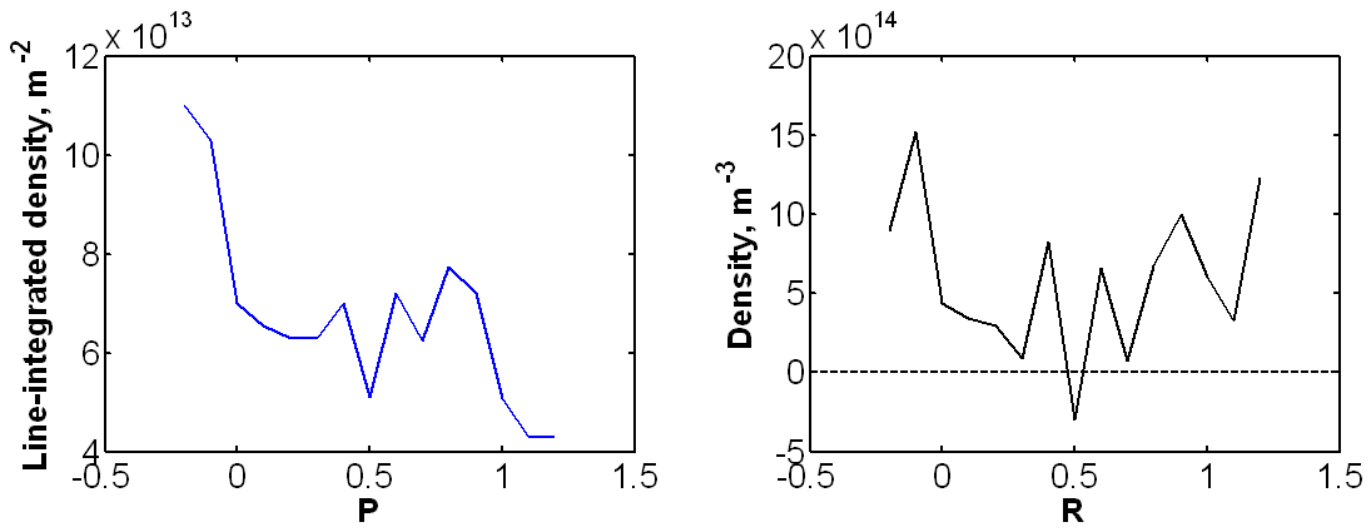

Figure 24. The line-integrated density plot and the corresponding boron density plot for the 6-kW Hall thruster operating at $600 \mathrm{~V}, 10 \mathrm{mg} / \mathrm{s}$.

From the figures of boron density, we can deduce that there was pretty much no detectable boron when the thruster was operating at $150 \mathrm{~V}, 10 \mathrm{mg} / \mathrm{s}$ and $150 \mathrm{~V}, 20 \mathrm{mg} / \mathrm{s}$. At $150 \mathrm{~V}, 30 \mathrm{mg} / \mathrm{s}$, the amount of boron is close to the minimum detectable threshold so the uncertainty is most likely high. The amount of uncertainty for all traces will be reported later in the sputter rate calculations. Note that for the $300 \mathrm{~V}, 20 \mathrm{mg} / \mathrm{s}$ condition, there were no usable traces at $\mathrm{P}=1.1$ and 1.2 due to a misalignment problem that was not caught until post-processing. The boron density is artificially set to 0 at those two data points so the amount of erosion for the $300 \mathrm{~V}, 20 \mathrm{mg} / \mathrm{s}$ condition may be slightly under-estimated (up to $10 \%$ based on data at other $300 \mathrm{~V}$ condition, which is less than the sensor uncertainty 
for this set). Also note that the density plot for the $600 \mathrm{~V}, 10 \mathrm{mg} / \mathrm{s}$ condition shown in Fig. 24 does not quite come down to zero at the edges of the measurement domain. This feature suggests the possibility that boron exists beyond $\mathrm{R}=-0.2$ and $\mathrm{R}=1.2$ at this operating condition. If this is true, the real sputtered rate is under-estimated in the measurement. However, the error is unlikely to be large because the geometry of the thruster does not really allow boron to show up far away from the channel. The uncertainty contributed is assumed to be smaller than the sensor uncertainty at this operating condition.

Figure 25 shows the sputtered boron flux as a function of non-dimensionalized radial position for the 300 and $600 \mathrm{~V}$ operating conditions. Flux plot for $150 \mathrm{~V}$ operating conditions are not shown since we already know the corresponding signals are close to or below the minimum detectable threshold of the sensor. Figure 25 shows a higher boron flux near the inner channel wall than near the outer channel wall. However, the respective area of boron emission is not the same due to changing radius. In comparison, Fig. 26 shows the sputtered boron particle rate (flux times area) of each onion ring for the same operating conditions. The total amount of boron being

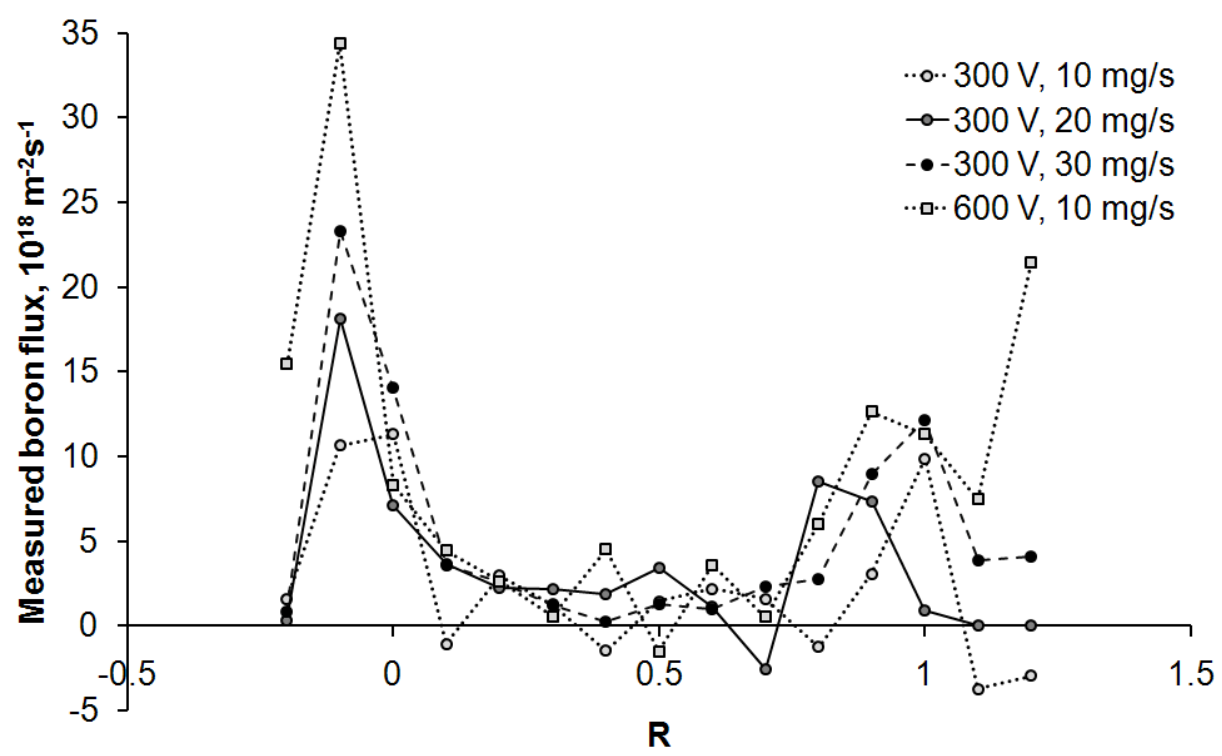

Figure 25. Boron flux as a function of non-dimensionalized radial position for the 6-kW Hall thruster operating at 300 and $600 \mathrm{~V}$ discharge voltage.

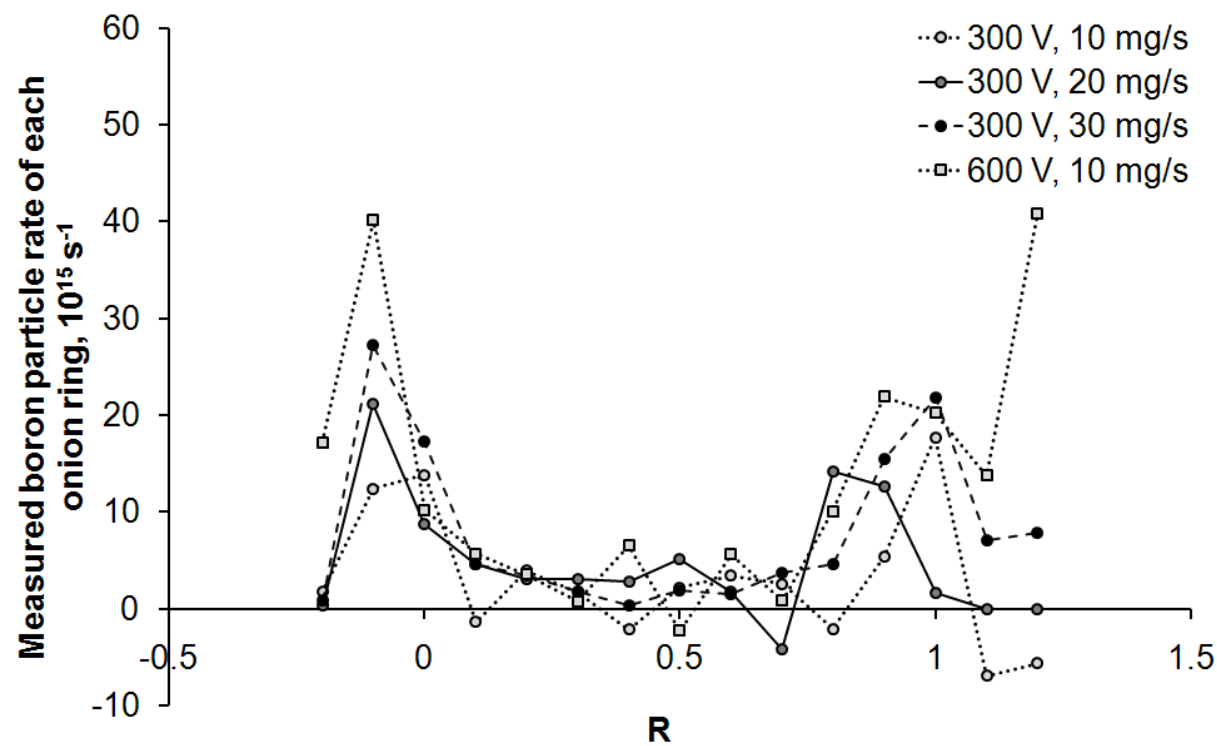

Figure 26. Boron particle rate of each onion ring as a function of non-dimensionalized radial position for the 6-kW Hall thruster operating at 300 and $600 \mathrm{~V}$ discharge voltage.

21

American Institute of Aeronautics and Astronautics 
sputtered off of the inner and outer channel walls appears to be about equal. However, these profiles may contain a large amount of error since the simple velocity model is used in the calculations. It is therefore difficult to draw any definitive conclusions regarding the relative amount of boron sputtered from one channel wall versus the other.

Figures 25 and 26 show that the detected boron is largely concentrated near the two walls. Recall the nominal wall radii are at $\mathrm{R}=0$ and $\mathrm{R}=1$. Due to enlargement of the channel at the exit plane, the actual radial location of the intersection between the two channel walls and the channel exit plane is at $R=-0.09$ and $R=1.09$, measured by profilometry. While the boron peak over the inner channel wall appears to be well defined, the boron peak over the outer channel wall is much more diffused and may be more susceptible to noise. It is unclear whether this difference is real or an artifact of noise.

\section{B. Relative and Absolute Sputter Rate}

Table 3 lists the relative and absolute channel wall material sputter rate calculated from the CRDS data for the 6$\mathrm{kW}$ Hall thruster at various operating conditions. This table shows how close the signals for the various $150 \mathrm{~V}$ conditions are to the minimum detectable threshold. The uncertainties of $62 \%$ and $100 \%$ for the $150 \mathrm{~V}, 10 \mathrm{mg} / \mathrm{s}$ and $150 \mathrm{~V}, 20 \mathrm{mg} / \mathrm{s}$ conditions, respectively, show that any signal present is drowned out by the noise. The minimum detectable sputter rate, shown in parentheses, is listed in place of the sputter rate for these two operating conditions. The true sputter rate is around or less than the value in parentheses.

Table 3. Relative and absolute sputter rate calculated from the CRDS data for the 6-kW Hall thruster.

\begin{tabular}{ccccccc}
\hline $\begin{array}{c}\text { Discharge } \\
\text { voltage, } \mathbf{V}\end{array}$ & $\begin{array}{c}\text { Anode mass } \\
\text { flow rate, } \mathbf{~ m g / s} \text { s }\end{array}$ & $\begin{array}{c}\text { Discharge } \\
\text { power, } \mathbf{~ k W}\end{array}$ & $\begin{array}{c}\text { Relative } \\
\text { sputter rate }\end{array}$ & $\begin{array}{c}\text { Associated } \\
\text { relative } \\
\text { uncertainty }\end{array}$ & $\begin{array}{c}\text { Sputter rate, } \\
\mathbf{m m}^{\mathbf{3} / \mathbf{s}}\end{array}$ & $\begin{array}{c}\text { Associated } \\
\text { relative } \\
\text { uncertainty }\end{array}$ \\
\hline 150 & 10 & 1.36 & 0.4 & $62 \%$ & $\left(3.7 \times 10^{-4}\right)$ & - \\
150 & 20 & 3.23 & -0.1 & $100 \%$ & $\left(5.7 \times 10^{-4}\right)$ & - \\
150 & 30 & 5.30 & 0.7 & $39 \%$ & $1.1 \times 10^{-3}$ & $63 \%$ \\
300 & 10 & 2.68 & 1.0 & $39 \%$ & $1.5 \times 10^{-3}$ & $63 \%$ \\
300 & 20 & 6.03 & 1.6 & $17 \%$ & $2.3 \times 10^{-3}$ & $53 \%$ \\
300 & 30 & 9.98 & 2.5 & $7 \%$ & $3.7 \times 10^{-3}$ & $51 \%$ \\
600 & 10 & 5.62 & 4.2 & $21 \%$ & $6.0 \times 10^{-3}$ & $54 \%$ \\
\hline
\end{tabular}

The relative sputter rate is normalized against the sputter rate for the $300 \mathrm{~V}, 10 \mathrm{mg} / \mathrm{s}$ operating condition. The sputter rate appears to rise with both discharge voltage and anode mass flow rate. In particular, doubling the discharge voltage from $300 \mathrm{~V}$ to $600 \mathrm{~V}$ while maintaining an anode mass flow rate of $10 \mathrm{mg} / \mathrm{s}$ roughly quadrupled the sputter rate. On the other hand, increasing the mass flow does not seem to have as dramatic of an effect. The 300 $\mathrm{V}, 20 \mathrm{mg} / \mathrm{s}$ and the $600 \mathrm{~V}, 10 \mathrm{mg} / \mathrm{s}$ operating conditions have almost the same discharge power but have very different relative sputter rates. The sputter rate appears to vary non-linearly with the discharge voltage and almost linearly with the anode mass flow rate.

\section{Comparing CRDS and Profilometry Results}

Table 4 compares the CRDS data to the profilometry results. To make this comparison, we multiplied the CRDS sputter rate by the amount of time spent at each operating condition and sum them up to obtain the total volume of BN ejected from the channel walls after 543 hours of thruster operation. The volume of ejected material is calculated from profilometry data by using geometry.

Profilometry is about seven times more accurate than CRDS. The CRDS measurement shows the right order of magnitude but is still off by $50 \%$. However, profilometry took much more operational time and resources to obtain results than CRDS did.

Several important observations can be made from the comparison in Table 4. First, the CRDS results appear to

Table 4. Comparison of total sputtered volume by diagnostics.

*Calculated using standard error propagation from a spatial measurement uncertainty of $0.05 \mathrm{~mm}$.

**Calculated using standard error propagation from the uncertainty at each operating condition.

\begin{tabular}{lcc}
\hline Methodology & Total volume of sputtered BN, $\mathbf{~ m m}^{\mathbf{3}}$ & Relative uncertainty \\
\hline Profilometry at 543 hours & 8437 & $8 \%{ }^{*}$ \\
CRDS measurement & 4249 & $56 \%{ }^{* *}$ \\
\hline
\end{tabular}


under predict the ejected $\mathrm{BN}$ volume. Some possible reasons have already been discussed in Section IV.D and include poor velocity modeling, ionization of neutral boron before they are detected, boron that exits the thruster in $\mathrm{BN}$ molecular complex, and possible incompleteness in the scanned lineshape. Also, it is possible that the record of operating hours is incomplete as the timing record is only accurate to a few minutes per entry. With that said, the total uncertainty in the operating hours should be no more than 10 hours or $2 \%$.

Another major possibility is that the erosion rate has changed between when the thruster was fresh and when it achieved 543 hours of operation. Erosion rate data from the SPT- $100^{31}$ replicated in Fig. 27 clearly show a rapid decrease in erosion rate during the first 800 hours of thruster operation. It would therefore not be surprising if a similar phenomenon is happening in the 6-kW thruster thus giving rise to under-prediction when using the CRDS data at 543 hours to calculate the total ejected volume of $\mathrm{BN}$. A more rigorous study involving taking CRDS and profilometry data at short regular intervals is needed to ascertain the

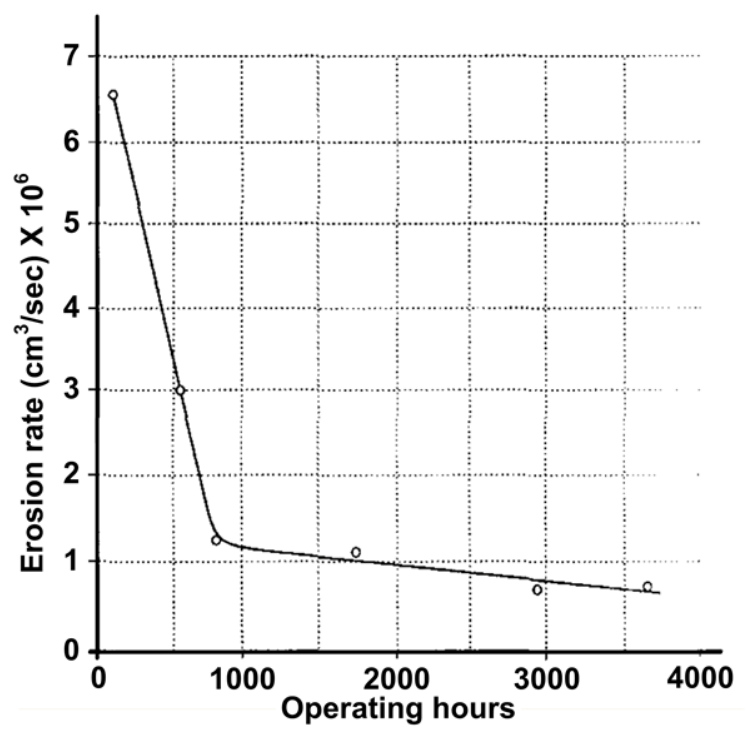

Figure 27. Erosion rate versus operating hours for the SPT-100. ${ }^{31}$ influence of the above phenomenon and to validate the use of CRDS for absolute erosion rate measurement.

With all of the above said, the authors believe the results shown in Table 4 are a very good sign. The fact that CRDS result is in the same order of magnitude as the profilometry results and the fact that we know many of the shortcomings and physical effects that can potentially account for the difference in results mean that CRDS really does have the potential to become a unique and highly accurate tool for studying Hall thruster channel wall erosion.

\section{Scaling Equation}

We will now study the relationship between the discharge power, anode mass flow rate, and sputter rate, by fitting the CRDS data to a general power-law form shown in Eq. (21),

$$
\mathrm{SR} \sim \mathrm{V}^{\mathrm{x}} \dot{\mathrm{m}}^{\mathrm{y}}
$$

where the discharge voltage, $\mathrm{V}$, is raised to the power of $\mathrm{x}$ and the anode mass flow rate, $\dot{\mathrm{m}}_{\mathrm{A}}$, is raised to the power of $y$. Note that this equation form has no dependence on the magnetic field so is only applicable if the magnetic field obeys certain scaling to the discharge voltage and anode mass flow rate. In the case of the 6-kW Hall thruster, the magnetic field is symmetric about the channel centerline and set to maximize the anode efficiency for a given operating condition. To find the value of $\mathrm{x}$, we perform power-law curve-fit on data from pairs of operating conditions having the same anode mass flow rate (i.e. 150 and $300 \mathrm{~V}$ at $30 \mathrm{mg} / \mathrm{s}$ and 300 and $600 \mathrm{~V}$ at $10 \mathrm{mg} / \mathrm{s}$ ). The value of $\mathrm{x}$ calculated this way is then averaged. The data from $150 \mathrm{~V}, 10$ and $20 \mathrm{mg} / \mathrm{s}$ conditions are not used because they do not represent positive boron detection. The value of $y$ is found by performing power-law curve-fit on the data from the $300 \mathrm{~V}$ operating conditions. Figure 28 shows the curve-fit for the coefficient x, and Fig. 29 shows the curve-fit for the coefficient $y$. The final result is shown in Eq. (22).

$$
\mathrm{SR} \sim \mathrm{V}^{1.9} \dot{\mathrm{m}}^{0.8}
$$

The exponent on the discharge voltage is found to be almost two. This is believed to be driven by a combination of the sputter yield and erosion zone length increasing with the discharge voltage. The increase in sputter yield with incident ion energy is predicted by a number of sputter yield models. Two of these models have been plotted for various angle-of-incidences (AOIs) in Fig. 30. The models predict that the rise in sputter yield is roughly linear with the ion energy to a first order estimate thus contributing a value of one to the exponent of the discharge voltage in Eq. (22). The other dominant contribution to the exponent on the discharge voltage, the increase in erosion zone length with discharge voltage, has been experimentally observed in a number of near-wall experiments, ${ }^{26,32}$ though this phenomenon is not well studied in the community due to a lack of experimental data. We can tentatively conclude that for the 6-kW Hall thruster over the tested operating conditions, the contribution of the erosion zone 


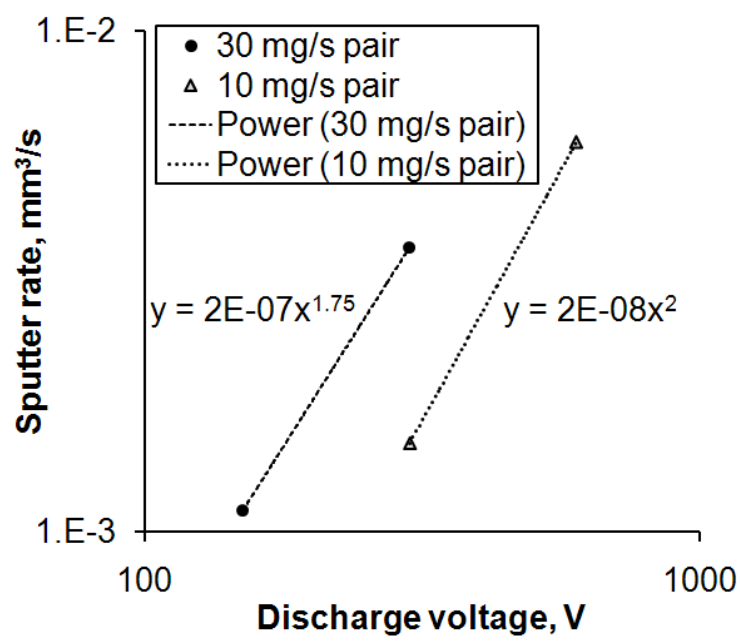

Figure 28. Curve-fit for the exponent of the discharge voltage in the relative erosion rate equation.

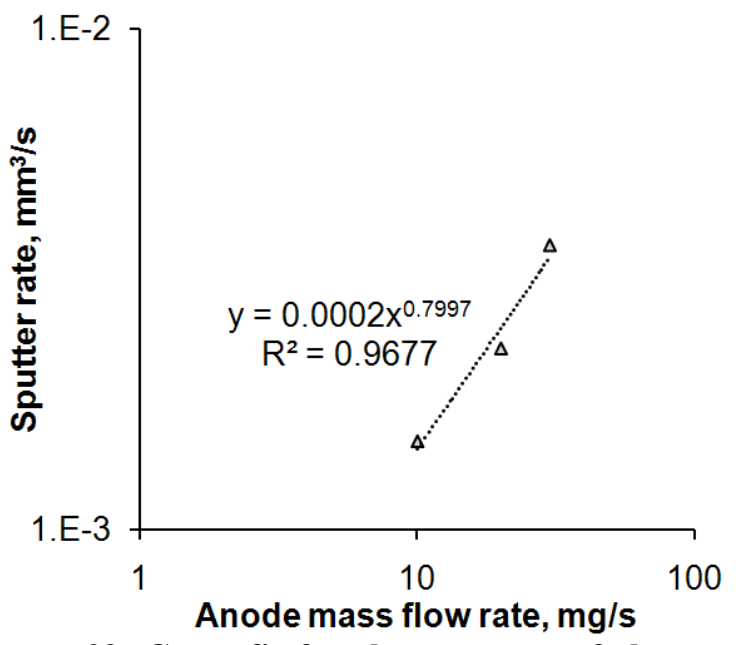

Figure 29. Curve-fit for the exponent of the anode mass flow rate in the relative erosion rate equation.

length effect to the exponent on the discharge voltage is roughly one. That is to say the erosion zone length scales linearly with the discharge voltage.

Interestingly, the curve-fit produced a value for the exponent of the anode mass flow rate at 0.8 , less than 1 , which implies that the sputter rate increases a bit less slowly than linear with the anode mass flow rate. This may provide evidence that the erosion zone is being pushed downstream as the anode mass flow rate increases. The uncertainty in the values of $\mathrm{x}$ and $\mathrm{y}$ is large and, due to the limited quantity of the data available, it is not meaningful to try to establish the value of the uncertainty. Furthermore, it is unclear how the scaling equation may be affected by higher order effects like plume divergence and presence of multiply-charged species. Nevertheless, the results show how the CRDS diagnostics can be used to obtain an engineering scaling equation that relates the sputter rate to the discharge voltage and anode mass flow rate.

\section{Application Ideas}

This section is included in this paper to describe other ways to utilize CRDS density measurement besides

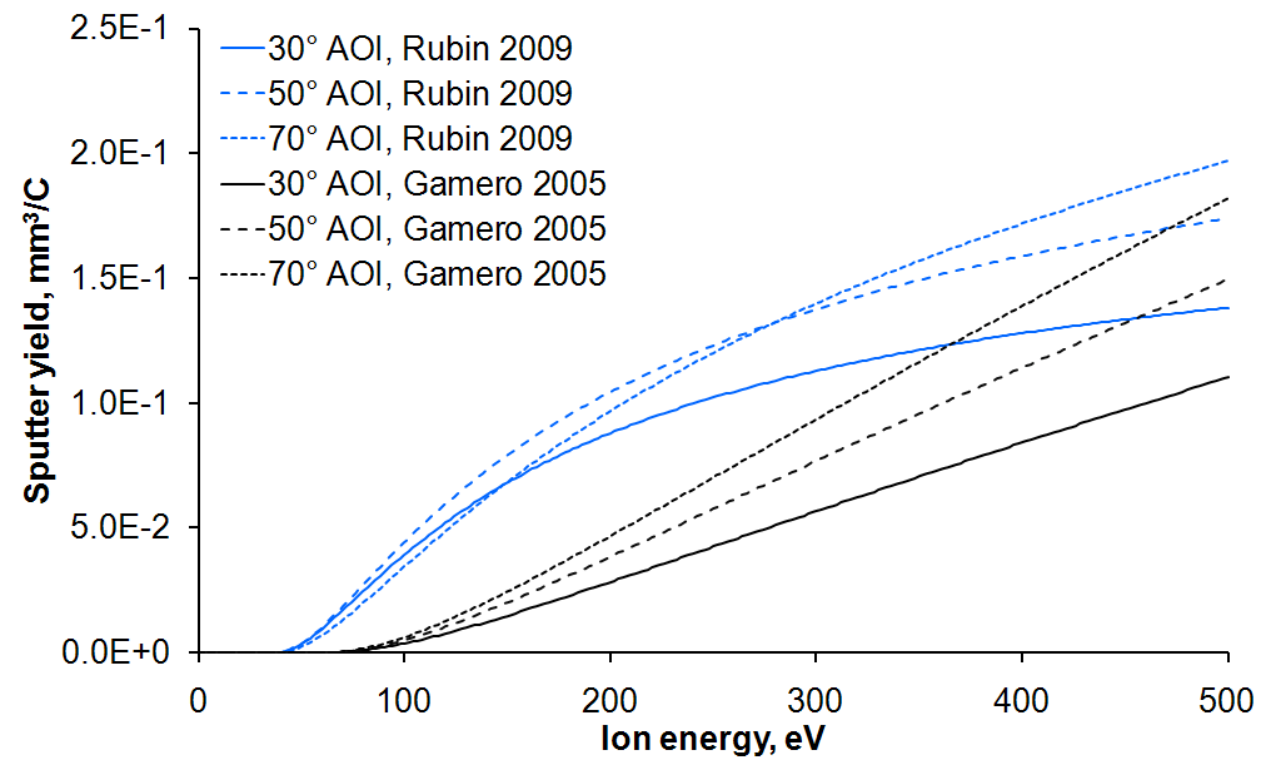

Figure 30. Sputter yield versus ion energy for two sputtering models at different angles of incidence.

Rubin 2009 refers to the model described in work by Rubin, et al., ${ }^{33}$ and Gamero 2005 refers to the model described in Gamero and Katz. ${ }^{34}$ 
parametric scientific study. It is mainly written to share ideas.

The ultimate goal of studying the channel wall erosion problem is to develop predictive tools that can aid in the design of long life Hall thrusters. The purpose of performing CRDS measurement is to obtain data that can help such development. However, it may be possible to use CRDS results itself as a predictive tool. Two applications of CRDS data for flight qualification come to mind.

The first application is to use the CRDS data to project the life of the channel walls from one operating condition to another. By performing boron density measurements in near-real-time, a thruster that has been qualified can potentially be re-qualified for another operating condition depending on how much difference there is between the conditions. At the very least, one can establish upper engineering limits for how much erosion to expect at the new operating condition.

The second application is to use the CRDS data with LIF measurements to perform accelerated life test. For example, we begin by obtaining data on the bombarding particles and the erosion rate for thruster $\mathrm{X}$, then use that data to make a short time step ( $\sim 500$ hours) prediction of what the channel walls will become. We now manufacture a new set of channel walls with the predicted profile and repeat the measurements. Depending how rapidly the walls are eroding, we can change the size of the time step. Five hundred hours of thruster operation would normally take 3 weeks. Since the CRDS and LIF data can be taken in 1-2 days once set up, the turnaround time for accelerated life test would only depend on how quickly new channels can be manufactured. As such, this approach may only work well if there is channel wall shaping capability on-site. However, even if it takes 1 week to shape a new ring, that will still be $3 \mathrm{x}$ time and operation resource savings. If the channel wall erosion rate drops over time, the time step can be made longer and the savings will increase. Of course, this approach is not a replacement for full-flight qualification, which tests other components besides the channel walls, but it is a more cost-efficient way to requalify the channel walls or just to study how the erosion of the channel walls progress.

\section{Conclusion and Future Works}

We have successfully demonstrated the first use of boron CRDS to study channel wall erosion in a Hall thruster environment to measure the channel wall erosion rate. Like all novel diagnostics, the first batch of data contains large amount of uncertainty, but the major sources of uncertainty have been identified. This experiment clearly demonstrated the potential of CRDS for obtaining in-situ near-real-time sputter rate measurement for studying the Hall thruster channel wall erosion problem. It further demonstrated how a simple engineering equation can be formulated to correlate relative erosion rate across different discharge voltages and anode mass flow rates for the test article.

There are many areas of possible improvement for the use of CRDS to study Hall thruster channel wall erosion, most of which relates to the sources of uncertainty previously described in the paper. The specific areas of improvement will be discussed in a future paper. For this paper, it is sufficient to say that the near-term focus of CRDS research should be on suppressing the degradation of the mirrors, improving the sensor sensitivity, and improving the boron velocity model.

In terms of future scientific studies, two avenues of investigation would be very helpful in continuing the research described in this paper. First, it is imperative that more data be collected at different high-voltage operating conditions $(300+\mathrm{V})$ because the creation of a better engineering scaling relationship for erosion requires more highvoltage data. This need was demonstrated in this paper when an attempt was made to create such a scaling relationship.

Second, more studies of the type described in this dissertation should be performed on various thrusters and at various stages of life. By comparing the erosion data across different thrusters we can determine whether the scaling relationship for predicting erosion rate at different operating conditions is universal across thrusters. By comparing the erosion data across different stages of life for the Hall thruster, we can determine whether and how the erosion physics changes as the channel erodes. These data will be very useful for developing models and design tools for Hall thrusters.

In addition, a wide variety of scientific investigations has now been enabled by a developed boron CRDS erosion diagnostic that was previously impractical to perform. For example, we can now measure the channel wall erosion rate as a function of changing magnetic field topology in an attempt to search for a topology that minimizes erosion rate (as opposed to maximizes performance). We can study the effect of different wall materials (this may require multiple lasers and mirror sets) to identify materials that are optimal from an erosion standpoint. There is a vast number of possible scientific ventures now available for exploring.

Furthermore, it would be interesting and highly valuable to Hall thruster erosion research to investigate how exactly the Hall thruster magnetic circuit erodes once exposed to plasma. The community has repeated cited the 
erosion of the channel walls and subsequent exposure of the magnetic circuit to plasma as a primary failure mode in the Hall thruster. However, little is known about the duration of time between the beginning of circuit exposure to plasma and actual failure of the thruster. A simple experiment to study the behavior of this failure mode could be performed by shaping the channel based on existing channel profiles such that the magnetic circuit is exposed.

Ultimately, the study of the Hall thruster channel wall erosion needs to be tied back to the basic underlying physics so that accurate, predictive models can be built to help future design works. In particular, a very important next step forward is to decipher the physical basis by which the erosion zone length increases with the discharge voltage. There is also the important question of how the erosion zone length changes with the magnetic field topology. Understanding these mechanisms are the keys to creating a model that can correlate erosion rate across different operating conditions for a Hall thruster, and eventually will lead to a model for predicting absolute erosion rate. 


\section{Acknowledgments}

The authors would like to acknowledge and thank Dr. Mitat Birkan of the Air Force Office of Scientific Research (AFOSR) for the DURIP grant, number FA9550-07-1-0535. The first author would like to acknowledge and thank Dr. Hani Kamhawi and the Glenn Research Center for support via the NASA Graduate Student Researchers Program, grant number NNX09AK99H.

\section{References}

${ }^{1}$ Peterson, P. Y. and Manzella, D. H., "Investigation of the Erosion Characteristics of a Laboratory Hall Thruster", 39th AIAA/ASME/SAE/ASEE Joint Propulsion Conference \& Exhibit, AIAA-2003-5005, Huntsville, AL, 20-23 Jul., 2003.

${ }^{2}$ Misuri, T., Milani, A., and Andrenucci, M., "Development of a Telemicroscopy Diagnostic Apparatus and Erosion Modelling in Hall Effect Thrusters", 31st International Electric Propulsion Conference, 2009-036, Ann Arbor, MI, 20-24 Sep., 2009.

${ }^{3}$ Pagnon, D., Balika, L., and Pellerin, S., "QCM and OES: two ways used to study simultaneously HET thruster chamber ceramic erosion. First QCM results on PPS100-ML validate previous OES measurements." 31st International Electric Propulsion Conference, 2009-118, Ann Arbor, MI, 20-24 Sep., 2009.

${ }^{4}$ Rubin, B., Topper, J. L., Farnell, C. C., and Yalin, A. P., "Quartz crystal microbalance-based system for high-sensitivity differential sputter yield measurements", Review of Scientific Instruments, Vol. 80, No. 10, doi:10.1063/1.3249560, 26 Oct., 2009, pp. 103506.

${ }^{5}$ Hargus, W. A., Jr. and Strafaccia, J., "Optical Boron Nitride Insulator Erosion Characterization of a 200 W Xenon Hall Thruster", 41st AIAA/ASME/SAE/ASEE Joint Propulsion Conference, AIAA-2005-3529, Tucson, AZ, 11-13 Jul., 2005.

${ }^{6}$ Garnier, Y., Viel, V., Roussel, J. F., and Bernard, J., "Low-energy xenon ion sputtering of ceramics investigated for stationary plasma thrusters", Journal of Vacuum Science and Technology, Vol. 17, No. 6, doi:10.1116/1.582050, Nov/Dec, 1999, pp. 32463254.

${ }^{7}$ Yalin, A. P., Surla, Y., Farnell, C., Butweiller, M., and Williams, J. D., "Sputtering Studies of Multi-Component Materials by Weight Loss and Cavity Ring-Down Spectroscopy", 42nd AIAA/ASME/SAE/ASEE Joint Propulsion Conference \& Exhibit, AIAA-2006-4338, Sacramento, CA, 9-12 Jul., 2006.

${ }^{8}$ Rubin, B., Topper, J. L., and Yalin, A. P., "Total and Differential Sputter Yields of Boron Nitride Measured by Quatz Crystal Microbalance and Weight Loss", 30th International Electric Propulsion Conference, 2007-074, Florence, Italy, $17-20$ Sep., 2007. ${ }^{9}$ Surla, V., Wilbur, P. J., Johnson, M., Williams, J. D., and Yalin, A. P., "Sputter erosion measurements of titanium and molybdenum by cavity ring-down spectroscopy", Review of Scientific Instruments, Vol. 75, No. 9, doi:10.1063/1.1786354, Sep., 2004, pp. 3025.

${ }^{10}$ Yamamoto, N., Tao, L., Rubin, B., Williams, J. D., and Yalin, A. P., "Sputter Erosion Sensor for Anode Layer-Type Hall Thrusters Using Cavity Ring-Down Spectroscopy", Journal of Propulsion and Power, Vol. 26, No. 1, Jan-Feb, 2010, pp. 142148.

${ }^{11}$ Yalin, A. P., et al., "High-Sensitivity Boron Nitride Sputter Erosion Measurements by Continuous-Wave Cavity Ring-Down Spectroscopy", 44th AIAA/ASME/SAE/ASEE Joint Propulsion Conference \& Exhibit, AIAA-2008-5091, Hartford, CT, 21-23 Jul., 2008.

${ }^{12}$ Tao, L., Lee, B., Yalin, A., Yamamoto, N., and Gallimore, A. D., "Development of a Cavity Ring-Down Spectroscopy Sensor for Boron Nitride Erosion in Hall Thrusters", 31st International Electric Propulsion Conference, 2009-146, Ann Arbor, MI, 2024 Sep., 2009.

${ }^{13}$ Berden, G., Peeters, R., and Meijer, G., "Cavity ring-down spectroscopy: Experimental schemes and applications", International Reviews in Physical Chemistry, Vol. 19, No. 4, 2000, pp. 565-607.

${ }^{14}$ Fuhr, J. R. and Wiese, W. L., "Tables of Atomic Transition Probabilities for Beryllium and Boron", Journal of Physical and Chemical Reference Data, Vol. 39, No. 1, doi:10.1063/1.3286088, 12 Mar., 2010, pp. 013101.

${ }^{15}$ Hargus, W. A., Jr. and Charles, C. S., "Near Plume Laser Induced Fluorescence Velocity Measurements of a 600 W Hall Thruster", 44th AIAA/ASME/SAE/ASEE Joint Propulsion Conference \& Exhibit, AIAA-2008-5004, Hartford, CT, 21-23 Jul., 2008.

${ }^{16}$ Dasch, C. J., "One-dimensional tomography: a comparison of Abel, onion-peeling, and filtered backprojection methods", Applied Optics, Vol. 31, No. 1, doi:10.1364/AO.31.001146, 1992, pp. 1146.

${ }^{17}$ Hanson, K. M., A Bayesian approach to nonlinear inversion: Abel inverison from x-ray data, Transport Theory, Invariant Imbedding, and Integral Equations, Lecture Notes in Pure and Applied Mathematics, edited by P. Nelson, et al., Vol. 115, Marcel Dekker, New York, 1989, 363-368.

${ }^{18}$ Saleh, B. E. A. and Teich, M. C., Resonator Optics, in Fundamentals of Photonics, Ch. 10, John Wiley \& Sons, Hoboken, NJ, 2007.

${ }^{19}$ Kogelnik, H. and Li, T., "Laser Beams and Resonators", Applied Optics, Vol. 5, No. 10, doi:10.1364/AO.5.001550, Oct., 1966, pp. $1550-1567$.

${ }^{20}$ Sigmund, P. i., in Sputtering by Particle Bombardment I, Ch., R. Behrisch, Editor, Springer, Berlin, 1981, pp. 9.

${ }^{21}$ Betz, G. and Wien, K., "Energy and angular distributions of sputtered particles", International Journal of Mass Spectrometry and Ion processes, Vol. 140, No. 1, doi:10.1016/0168-1176(94)04052-4, Dec., 1994, pp. 1-110.

${ }^{22}$ Tao, L. and Yalin, A., "LIF Velocity Measurement of Sputtered Boron Atoms from Boron Nitride Target", 46th AIAA/ASME/SAE/ASEE Joint Propulsion Conference \& Exhibit, AIAA-2010-6526, Nashville, TN, 25-28 Jul., 2010. 
${ }^{23}$ Lehmann, K. K., Berden, G., and Engeln, R., An Introduction to Cavity Ring-Down Spectroscopy, in Cavity Ring-Down Spectroscopy, Ch., G. Berden and R. Engeln, Editors, Wiley, Chippenham, U.K., 2009.

${ }^{24}$ Yalin, A. P., Surla, V., Butweiller, M., and Williams, J. D., "Detection of sputtered metals with cavity ring-down spectroscopy", Applied Optics, Vol. 44, No. 30, Oct., 2005, pp. 6496-6505.

${ }^{25}$ Dutouquet, C., Acquaviva, S., and Hermann, J., "Detection of boron nitride radicals by emission spectroscopy in a laserinduced plasma", Spectrochimica Acta Part B, Vol. 56, No. 6, doi:10.1016/S0584-8547(01)00188-4, 13 Jul., 2001, pp. 629-635.

${ }^{26}$ Huang, W., Gallimore, A. D., and Smith, T. B., "Two-Axis Laser-Induced Fluorescence of Singly-Charged Xenon inside a 6kW Hall Thruster", 49th AIAA Aerospace Sciences Meetings, AIAA-2011-1015, Orlando, FL, 4-7 Jan., 2011.

${ }^{27}$ Rubin, B., Topper, J. L., and Yalin, A. P., "Total and differential sputter yields of boron nitride measured by quartz crystal microbalance", Journal of Physics D: Applied Physics, Vol. 42, No. 20, doi:10.1088/0022-3727/42/20/205205, 23 Sep., 2009 , pp. 205205.

${ }^{28}$ Kim, Y.-K. and Stone, P. M., "Ionization of boron, aluminum, gallium, and indium by electron impact", Physical Review A, Vol. 64, No. 5, doi:10.1103/PhysRevA.64.052707, 4 Oct., 2001, pp. 052707.

${ }^{29}$ Reid, B. M., "The Influence of Neutral Flow Rate in the Operation of Hall Thrusters", Ph.D. Dissertation, Aerospace Engineering, University of Michigan, Ann Arbor, MI, 2008.

${ }^{30}$ Yalin, A. P. and Surla, V., "Velocity measurements by cavity ringdown spectroscopy", Optics Letters, Vol. 30, No. 23, Dec., 2005, pp. 3219-3221.

${ }^{31}$ Absalamov, S. K., et al., "Measurement of Plasma Parameters in the Stationary Plasma Thruster (SPT-100) Plume and Its Effect on Spacecraft Components", 28th AIAA/ASME/SAE/ASEE Joint Propulsion Conference, AIAA-1992-3156, Nashville, TN, 6-8 Jul., 1992.

${ }^{32}$ Shastry, R., "Experimental Characterization of the Near-Wall Region in Hall Thrusters and its Implications on Performance and Lifetime", Ph.D. Dissertation, Aerospace Engineering, University of Michigan, Ann Arbor, MI, 2011.

${ }^{33}$ Rubin, B., Topper, J. L., and Yalin, A. P., "Total and Differential Sputter Yields of Boron Nitride Measured by Quartz Crystal Microbalance", 31st International Electric Propulsion Conference, 2009-042, Ann Arbor, MI, 20-24 Sep., 2009.

${ }^{34}$ Gamero-Castano, M. and Katz, I., "Estimation of Hall Thruster Erosion using HPHall", 29th International Electric Propulsion Conference, IEPC 2005-303, Princeton, NJ, 31 Oct. - 4 Nov., 2005. 